\title{
$17 \beta$-Estradiol and estrogen receptor $\alpha$ protect right ventricular function in pulmonary hypertension via BMPR2 and apelin
}

\author{
Andrea L. Frump, ${ }^{1}$ Marjorie Albrecht, ${ }^{1}$ Bakhtiyor Yakubov, ${ }^{1}$ Sandra Breuils-Bonnet, ${ }^{2}$ Valérie Nadeau, ${ }^{2}$ Eve Tremblay, ${ }^{2}$ \\ Francois Potus, ${ }^{2}$ Junichi Omura, ${ }^{2}$ Todd Cook, ${ }^{1}$ Amanda Fisher, ${ }^{1}$ Brooke Rodriguez, ${ }^{1}$ R. Dale Brown, ${ }^{3}$ Kurt R. Stenmark, ${ }^{3}$ \\ C. Dustin Rubinstein, ${ }^{4}$ Kathy Krentz, ${ }^{4}$ Diana M. Tabima, ${ }^{5}$ Rongbo Li, ${ }^{6}$ Xin Sun, ${ }^{6}$ Naomi C. Chesler, ${ }^{5}$ Steeve Provencher, ${ }^{2}$ \\ Sebastien Bonnet, ${ }^{2}$ and Tim Lahm ${ }^{1,7,8}$
}

'Department of Medicine, Division of Pulmonary, Critical Care, Sleep and Occupational Medicine, Indiana University School of Medicine, Indianapolis, Indiana, USA. ${ }^{2}$ Pulmonary Hypertension Research Group, Institute Universitaire de Cardiologie et de Pneumologie de Québec - Université Laval, Quebec City, Quebec, Canada. ${ }^{3}$ Department of Pediatrics, University of Colorado-Denver, Aurora, Colorado, USA. ${ }^{4}$ Cenome Editing and Animal Models Core, University of Wisconsin Biotechnology Center, ${ }^{5}$ Department of Biomedical Engineering, and ${ }^{6}$ Department of Cenetics, University of Wisconsin-Madison, Madison, Wisconsin, USA. 'Department of Anatomy, Cell Biology \& Physiology, Indiana University School of Medicine, Indianapolis, Indiana, USA. ${ }^{8}$ Richard L. Roudebush Veterans Affairs Medical Center, Indianapolis, Indiana, USA

Women with pulmonary arterial hypertension (PAH) exhibit better right ventricular (RV) function and survival than men; however, the underlying mechanisms are unknown. We hypothesized that 17ק-estradiol (E2), through estrogen receptor $\alpha$ (ER- $\alpha$ ), attenuates PAH-induced RV failure (RVF) by upregulating the procontractile and prosurvival peptide apelin via a BMPR2-dependent mechanism. We found that ER- $\alpha$ and apelin expression were decreased in RV homogenates from patients with RVF and from rats with maladaptive (but not adaptive) RV remodeling. RV cardiomyocyte apelin abundance increased in vivo or in vitro after treatment with E2 or ER- $\alpha$ agonist. Studies employing ER- $\alpha$-null or ER- $\beta$-null mice, ER- $\alpha$ loss-of-function mutant rats, or siRNA demonstrated that ER- $\alpha$ is necessary for E2 to upregulate RV apelin. E2 and ER- $\alpha$ increased BMPR2 in pulmonary hypertension RVs and in isolated RV cardiomyocytes, associated with ER- $\alpha$ binding to the Bmpr2 promoter. BMPR2 is required for E2-mediated increases in apelin abundance, and both BMPR2 and apelin are necessary for E2 to exert RV-protective effects. E2 or ER- $\alpha$ agonist rescued monocrotaline pulmonary hypertension and restored RV apelin and BMPR2. We identified what we believe to be a novel cardioprotective E2/ER- $\alpha$ /BMPR2/apelin axis in the RV. Harnessing this axis may lead to novel RV-targeted therapies for PAH patients of either sex.

\section{Introduction}

Pulmonary arterial hypertension (PAH) is characterized by dysregulated vasoconstriction, muscularization of precapillary arteries, and formation of complex obstructive plexiform lesions in the pulmonary vasculature (1). This leads to increased pulmonary vascular resistance and right ventricular (RV) strain, eventually resulting in the development of RV failure (RVF, ref. 1). Current therapies target vasodilatory pathways in the lung but are not curative (2). Given that RV function is the main determinant of survival in $\mathrm{PAH}$, recent guidelines have called for the development of $\mathrm{RV}$-specific therapies $(3,4)$. To develop targets for such RV-specific therapies, it is critical to identify modifiers of RV function in PAH.

Even though PAH is sexually dimorphic and more common in women, female PAH patients survive longer than male

Conflict of interest: ALF's institution received research funding from Actelion. SB served on advisory boards for Actelion, Janssen, and Resverlogix. TL has received consultancy fees from Bayer and Altavant Sciences and was the site principal investigator for a clinical trial funded by Complexa.

Copyright: ( 2021, American Society for Clinical Investigation.

Submitted: April 8, 2019; Accepted: January 22, 2021; Published: March 15, 2021

Reference information: J Clin Invest. 2021;131(6):e129433.

https://doi.org/10.1172/JCl129433. patients $(5,6)$. This survival advantage has been attributed to better RV function in females, through as yet unknown mechanisms $(5,7,8)$. Female PAH patients exhibit better contractility, higher RV ejection fraction, and better RV-pulmonary arterial coupling than their male counterparts, and recent studies demonstrate that superior RV function contributes to the female survival advantage in PAH (8-10). Sex differences in RV function are mediated at least in part by biologically relevant effects of sex hormones, evidenced by correlations of estrogen levels with RV function in healthy postmenopausal hormone therapy users (7) and by higher cardiac indices in female PAH patients compared with male patients that are not observed in patients older than 45 years (11). These data suggest that to understand sex differences in PAH mortality, it is imperative to decipher the effects of sex hormones on the RV. Ultimately, a better understanding of the molecular mechanisms underlying superior female RV function would be expected to facilitate the development of nonhormonal and RV-specific therapies for patients with PAH and RVF of either sex.

We previously identified the sex steroid $17 \beta$-estradiol (E2) as a mediator of procontractile, antiinflammatory, and antiapoptotic effects in the RV $(12,13)$. These actions translated into improved $\mathrm{RV}$ function and exercise capacity without negatively affecting 
pulmonary vascular remodeling. Here, we aimed to decipher the yet unidentified mechanisms and downstream mediators of E2's RV-protective effects. We demonstrated that E2 exerts protective effects on RV function and maladaptive remodeling via estrogen receptor $\alpha(E R-\alpha)$, and we identified a mechanism by which ER- $\alpha$ activates BMPR2 signaling to upregulate apelin, a potent effector of cardiac contractility $(14,15)$. While BMPR2 and apelin are required for cardiac development and pulmonary vascular homeostasis (16-19), we now provide evidence that this pathway, via $\mathrm{ER}-\alpha$, is also functioning in the $\mathrm{RV}$, demonstrating a molecular basis for E2's RV-protective effects.

\section{Results}

Evidence of an ER- $\alpha$ /apelin signaling axis in human RV. E2 signals through 2 main receptors, ER- $\alpha$ and ER- $\beta$ (20). We previously showed that E2 and ER- $\alpha$ agonist treatment are RV protective in rat models of pulmonary hypertension-induced RVF and that ER- $\alpha$ abundance correlates with improved RV adaptation $(12,13)$. We therefore sought to identify molecular evidence of an E2/ER- $\alpha$ signaling axis in RVs from patients with and without RVF (21).

ER- $\alpha$ protein was decreased in RV homogenates from male and female patients with RVF compared with controls (Figure 1A). ER- $\beta$ expression, on the other hand, did not differ between groups (Supplemental Figure 1A; supplemental material available online with this article; https://doi.org/10.1172/JCI129433DS1). We localized ER- $\alpha$ to RV cardiomyocytes (RVCMs) as well as RV endothelial cells (RVECs; Figure 1, B and C). In RVCMs, we noted a decrease in abundance of nuclear and cytoplasmic ER- $\alpha$, whereas in RVECs, only cytoplasmic ER- $\alpha$ was decreased. No sex differences in ER- $\alpha$ or ER- $\beta$ expression were noted.

In previous studies, E2 repletion in sugen/hypoxia-induced $\mathrm{PH}(\mathrm{SuHx}-\mathrm{PH})$ rats was associated with increased expression of the procontractile and prosurvival peptide apelin (12). We therefore evaluated apelin expression in the human RV. We found that $\mathrm{RV}$ apelin protein abundance was decreased in RVF versus controls (Figure 2A). Interestingly, in patients with RVF, RV apelin abundance correlated positively with ER- $\alpha$ expression and cardiac output (Figure 2, B and C). Furthermore, apelin expression correlated negatively with RV fibrosis and RVCM hypertrophy (Figure 2, D and E). Similar to ER- $\alpha$, both apelin and its receptor APLNR localized to RVCMs and RVECs (Figure 2, F and G). Apelin and APLNR fluorescence intensity were significantly decreased in RVCMs and tended to be decreased in RVECs (Figure 2, B and C). No sex differences in apelin or APLNR expression were noted. No correlations were found between ER- $\beta$ expression and apelin abundance or cardiac output (Supplemental Figure 1, B and C). These data suggest an RV-protective ER- $\alpha$ /apelin signaling axis in human RVs, with decreased expression of ER- $\alpha$, apelin, and APLNR in the setting of RVF.

Apelin is decreased in RV characterized by maladaptive but not adaptive remodeling. To mechanistically study apelin's role in the $\mathrm{RV}$, we evaluated expression of apelin and its receptor in male rat RVs. Apelin and APLNR were strongly expressed in RVCMs and vascular cells; apelin was most strongly expressed in the vasculature and APLNR was expressed in the vasculature and cardiomyocytes (Figure 3A). To evaluate apelin's role in preventing the progression from adaptive to maladaptive RV remodeling, we measured its expression in RV homogenates from male rats with SuHx-PH, monocrotaline-induced $\mathrm{PH}$ (MCT-PH), and pulmonary artery banding (PAB) with either maladaptive RV hypertrophy (characterized by decreased cardiac output, fibrosis, and altered expression of genes involved in fatty acid metabolism and neurohormonal activation; Supplemental Figure 2) or adaptive RV hypertrophy (characterized by preserved cardiac output, lack of fibrosis, and maintained/less profoundly altered gene expression; Supplemental Figure 2). Apelin was decreased in maladaptive but not adaptive (Figure 3B) RV hypertrophy, suggesting protective effects of apelin against RVF development. Decreases in apelin were specific to the RV; no alterations in levels were noted in the left ventricle (LV; Supplemental Figure 3).

We next correlated RV apelin expression with mediators of RV structure and function (Figure 3C). RV apelin mRNA correlated negatively with increases in RVSP, RV hypertrophy, and proapoptotic signaling and positively with cardiac output in male and female rats. Finally, RVCMs isolated from male $\mathrm{SuHx}-\mathrm{PH}$ rats exhibited decreased apelin protein expression compared with controls (Figure 3D). These data suggest that decreased myocardial apelin is a hallmark of RVF and that apelin may mediate favorable RV adaptations.

RVCM-derived apelin promotes RVEC and RVCM function. Our immunolocalization studies (Figure 2) suggest that apelin could exert RV-protective effects by targeting RVCMs and/or RVECs. Beneficial effects of apelin in cardiac endothelial cells have been established $(22,23)$. However, the role of cardiomyocyte apelin is not well defined. Given the decreased expression of apelin and APLNR in RVCMs, we performed studies using conditioned media to delineate the role of RVCM apelin. RVCM apelin was knocked down by siRNA, the cell culture media collected, and naive RVECs and RVCMs exposed to the conditioned media (Figure 4, $\mathrm{A}$ and $\mathrm{B}$ ). Exposure to siRNA apelin-conditioned media resulted in decreased RVEC migration and tube formation (Figure 4, C and D) as well as decreased RVCM activation of the prosurvival mediator ERK1/2 (24) and decreased expression of the prosurvival and procontractile signaling mediator PKC- $\varepsilon$ (refs. 25, 16 and Figure 4, $\mathrm{E}$ and F). Similarly, treatment of RVECs or RVCMs with APLNR antagonist ML221 rendered these cells unresponsive to stimulatory effects of RVCM-conditioned media on migration, angiogenesis, and ERK1/2 activation and PKC- $\varepsilon$ expression (Supplemental Figure 4). These data demonstrated that cardiomyocyte apelin has physiologically relevant paracrine effects on RVECs and RVCMs.

E2 increases apelin expression in RVCMs. We next hypothesized that E2 upregulates apelin in RVCMs. Since inflammation and cardiomyocyte apoptosis are key features of $\operatorname{RVF}(26,27)$, we evaluated E2's effects on H9c2 cardiomyoblasts stressed with TNF- $\alpha$ or staurosporine (Figure 5, A and B). Interestingly, E2 pretreatment attenuated TNF- $\alpha$ - or staurosporine-induced decreases in apelin levels (Figure 5, A and B). In vivo, we found that ovariectomized SuHx-PH females replete with E2 exhibited $40 \%$ higher apelin expression in RV homogenates than ovariectomized SuHx-PH females without E2 (Figure 5C). In RVCMs, SuHx-PH-induced decreases in apelin were abrogated in cells isolated from male $\mathrm{SuHx}-\mathrm{PH}$ rats treated with E2 in vivo (Figure 5D). Finally, treatment of RVCMs isolated from male and female SuHx-PH or control rats with E2 in vitro increased apelin protein 1.9- and 3-fold, 
A

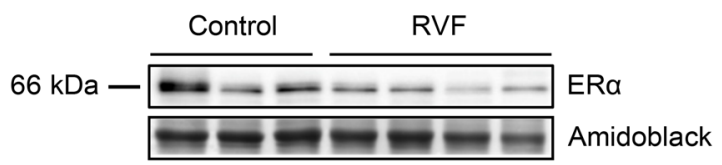

B

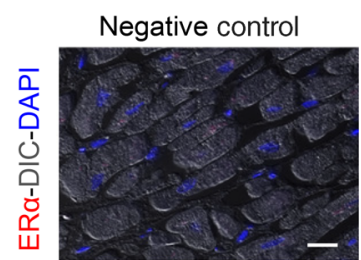

Human control RV
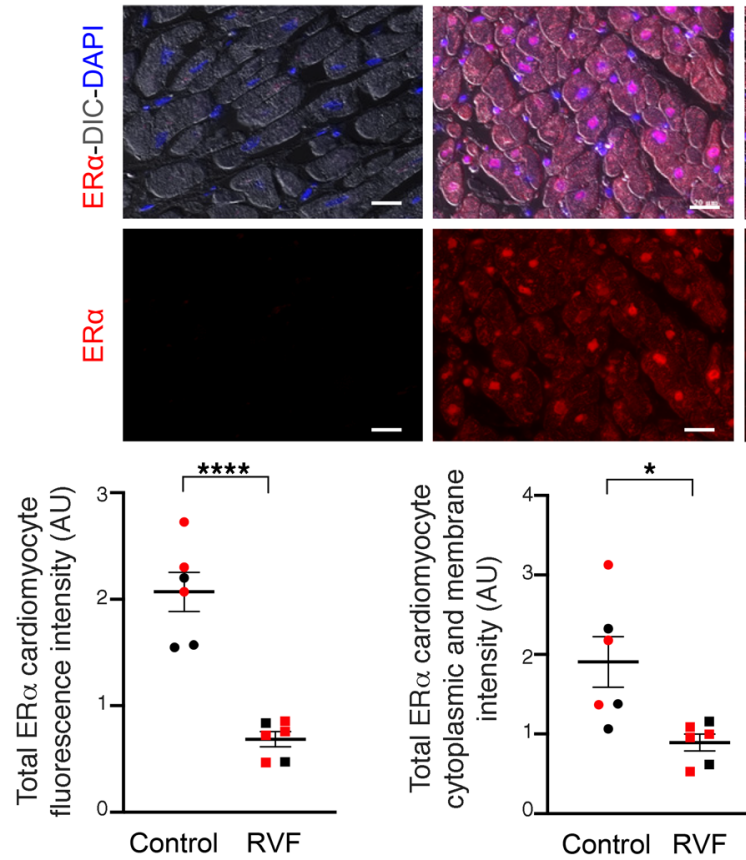

C

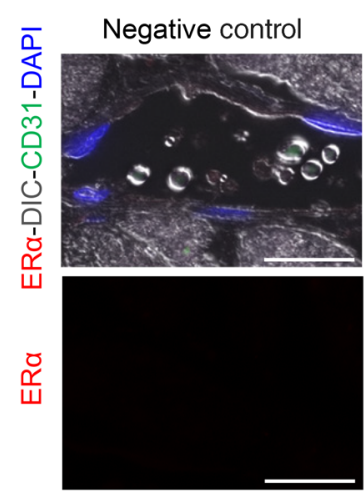

Human control RV
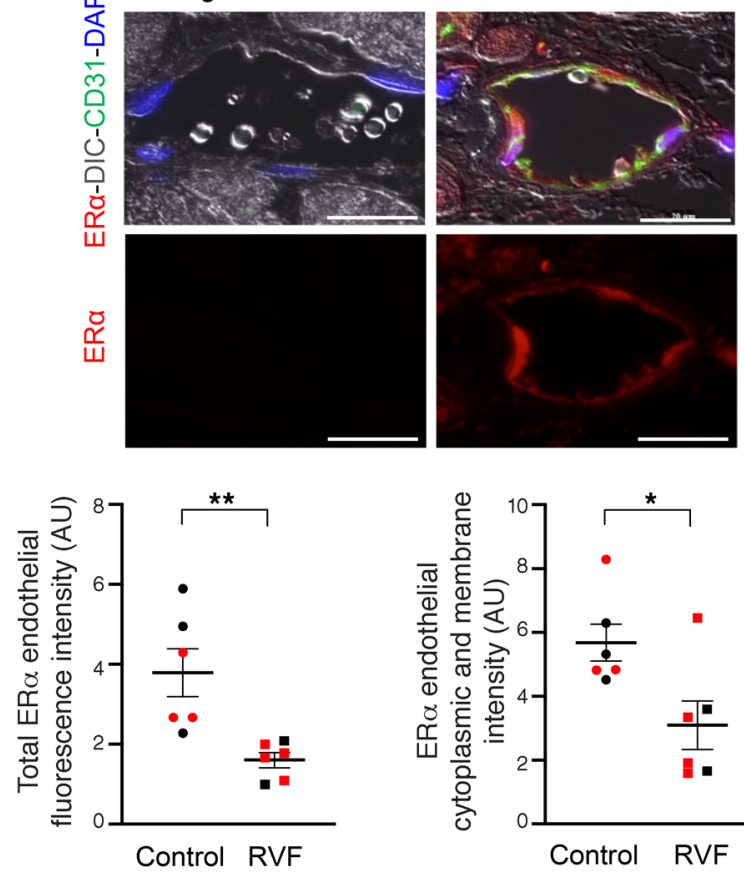

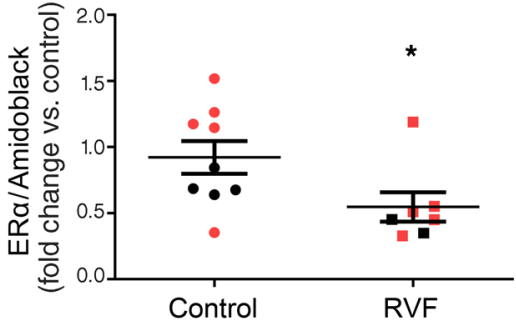

Human RVF
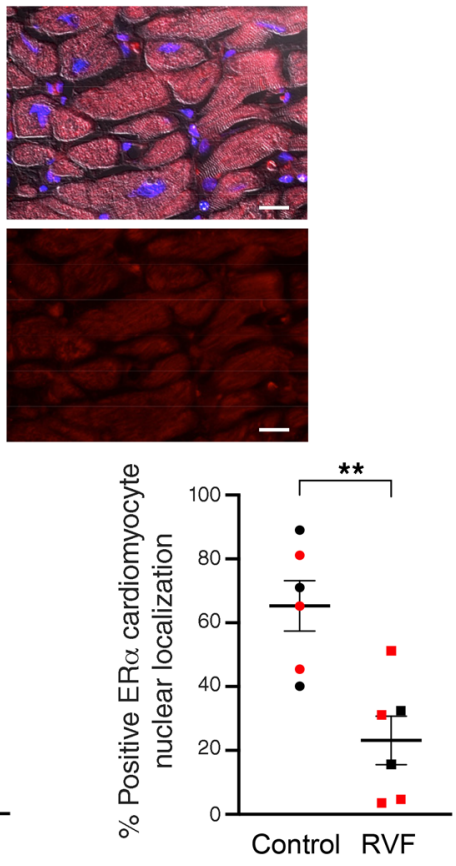

Human RVF
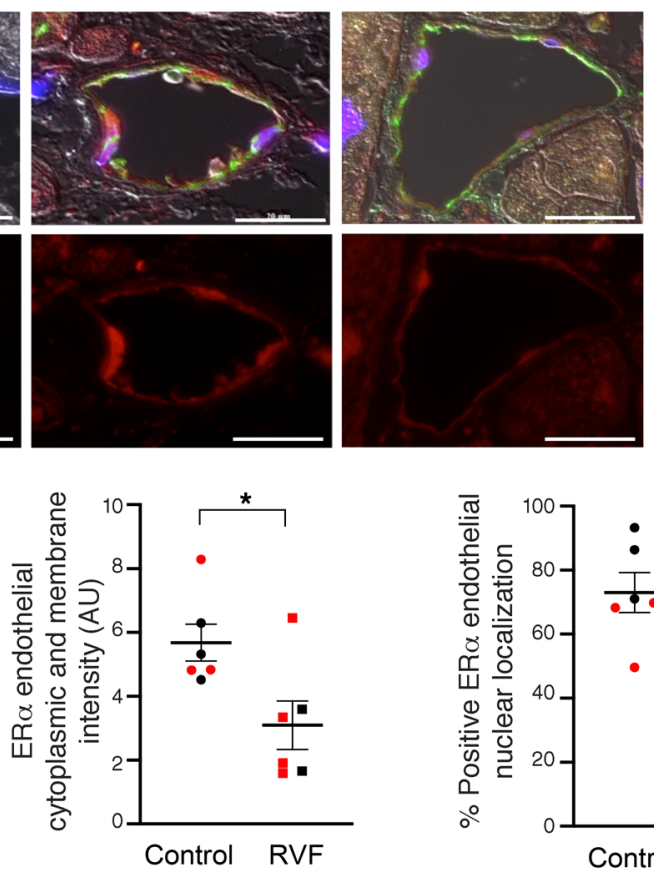

Figure 1. ER- $\alpha$ localizes to cardiomyocytes and endothelial cells in the human RV and is less abundant in patients with RV failure. (A) ER- $\alpha$ expression in human RV measured by Western blot. Quantification by densitometry shown on right. (B) Representative immunohistochemistry images of RV cardiomyocyte ER- $\alpha$ expression and localization in human control RVs and RVs from patients with RV failure (RVF). Cardiomyocyte localization was established by differential interference contrast (DIC). Quantification of total ER- $\alpha$ fluorescence intensity, cytoplasmic and membrane fluorescence intensity (measured in $\mathrm{AU}$ ), and nuclear localization (colocalization with DAPI) is shown in graphs. Images are at 20x magnification; scale bar: 20 $\mu \mathrm{m}$. (C) Representative immunocytochemistry images of ER- $\alpha$ expression and localization in RV endothelial cells in human control RVs and RVs from patients with RVF. Endothelial cell localization was established by colocalization with CD31. Total ER- $\alpha$ fluorescence intensity, cytoplasmic and membrane fluorescence intensity, and nuclear localization (colocalization with DAPI) are quantified in graphs. Images are at $40 \times$, scale bars: 20 $\mu \mathrm{m}$. Red and black symbols in graphs represent samples from female and male patients, respectively. Error bars represent mean \pm SEM. ${ }^{*} P<0.05,{ }^{* *} P$ $<0.01,{ }^{* * *} P<0.0001$ versus control by Student's $t$ test. 
A

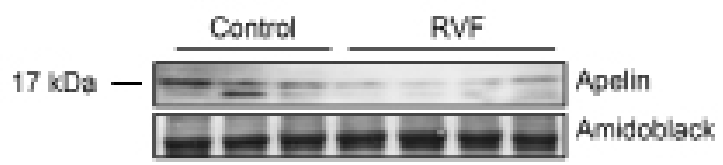

B

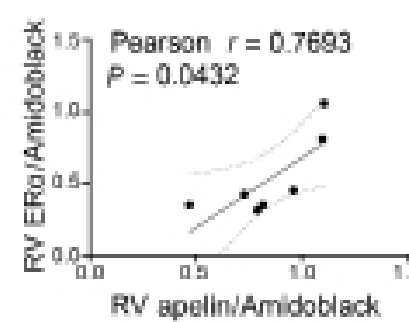

C

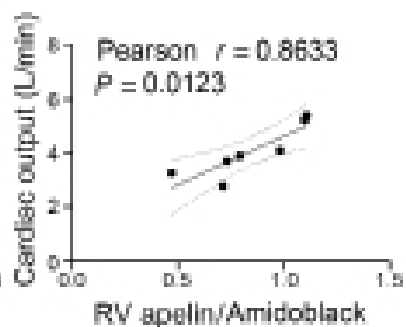

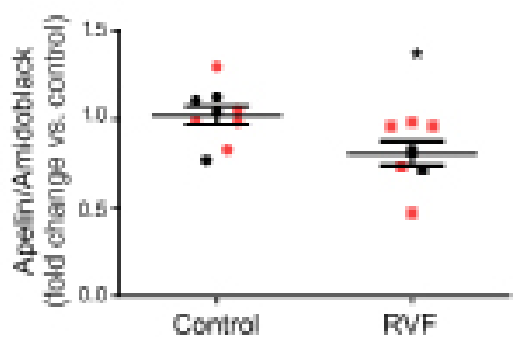

D

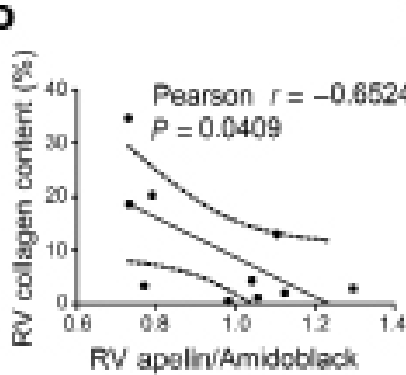

E

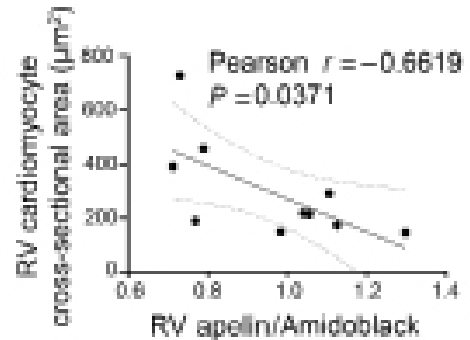

$\mathbf{F}$
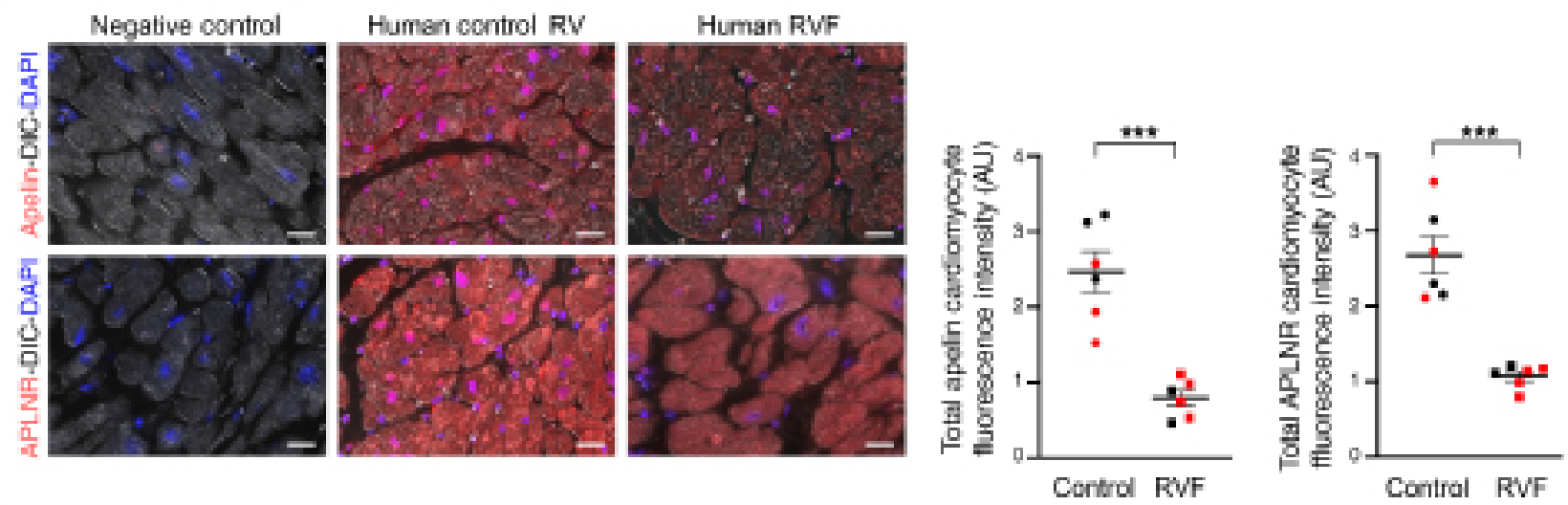

G
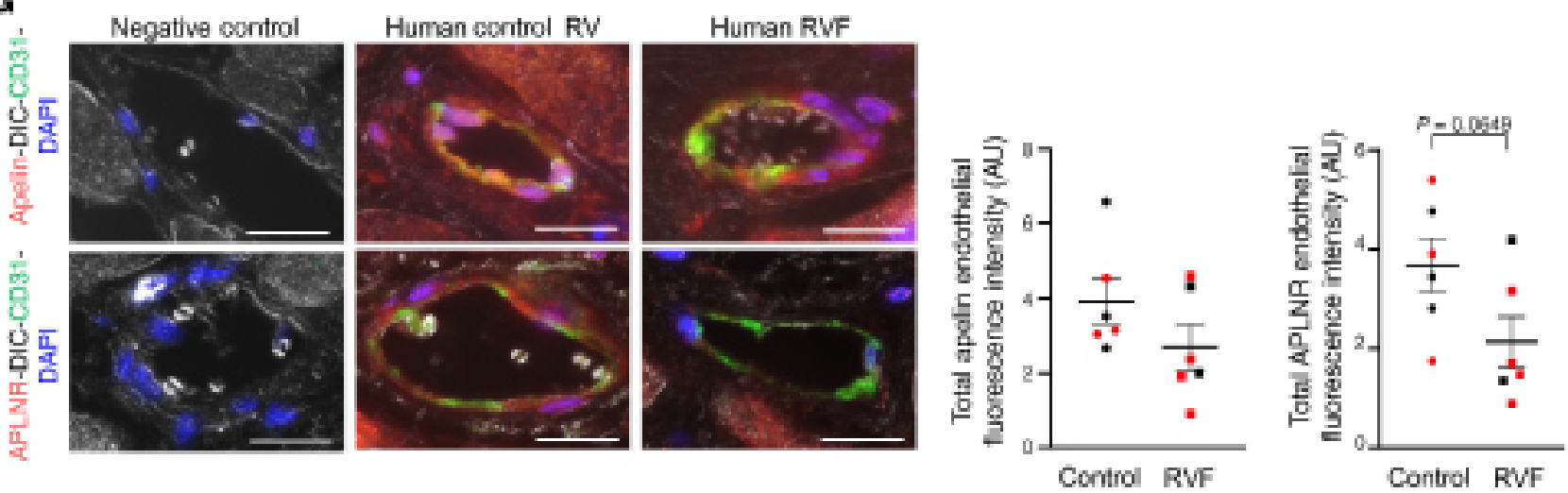

Figure 2. RV cardiomyocyte apelin and apelin receptor (APLNR) expression are decreased in patients with RV failure. (A) Apelin expression in human RV tissue measured by Western blot. Quantification by densitometry shown on right. (B-E) Apelin expression correlates positively with ER- $\alpha$ protein (B) and cardiac output (C) in RVs from patients with RV failure (RVF) and negatively with RV collagen content (D; expressed as percentage of RV cross-

section) and RV cardiomyocyte size (E; measured as cardiomyocyte cross-sectional area [CSA]) in human control RVs and RVs from RVF patients. Cardiac output data was available for RVF patients only. RV collagen content and RV cardiomyocyte size were analyzed in a randomly selected subgroup of control subjects. (F) Representative immunohistochemistry images of cardiomyocyte apelin and APLNR expression in human control RVs and RVs from patients with RVF. Cardiomyocyte localization was established by differential interference contrast (DIC). Cardiomyocyte apelin or APLNR fluorescence intensity is quantified in graphs. Images are at $20 \times$ magnification; scale bars: $20 \mu \mathrm{m}$. (C) Representative immunocytochemistry images of endothelial cell apelin and APLNR expression and localization in human control RVs and RVs from patients with RVF. Endothelial cell localization was established by colocalization with CD31. Apelin or APLNR fluorescence intensity is quantified in graphs. Images are at 40x, scale bars: $20 \mu \mathrm{m}$. Red and black symbols in graphs represent female and male samples, respectively. $(\mathbf{A}, \mathbf{F}$, and $\mathbf{G}){ }^{*} P<0.05,{ }^{* * *} P<0.001$ versus control by Student's $t$ test. Error bars represent mean \pm SEM. (B-E) Pearson's $R$ value and $P$ value shown. Dashed lines represent $95 \% \mathrm{CI}$. 
A

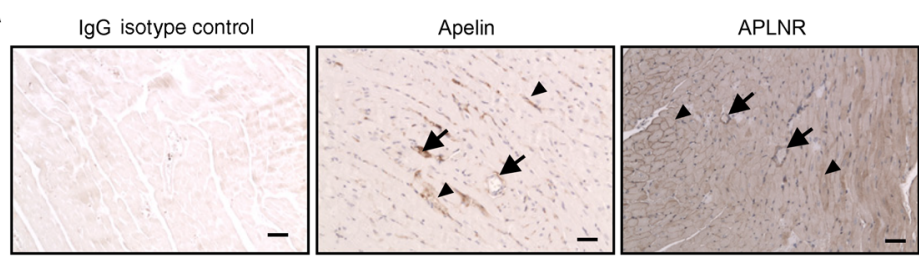

B

Adaptive remodeling
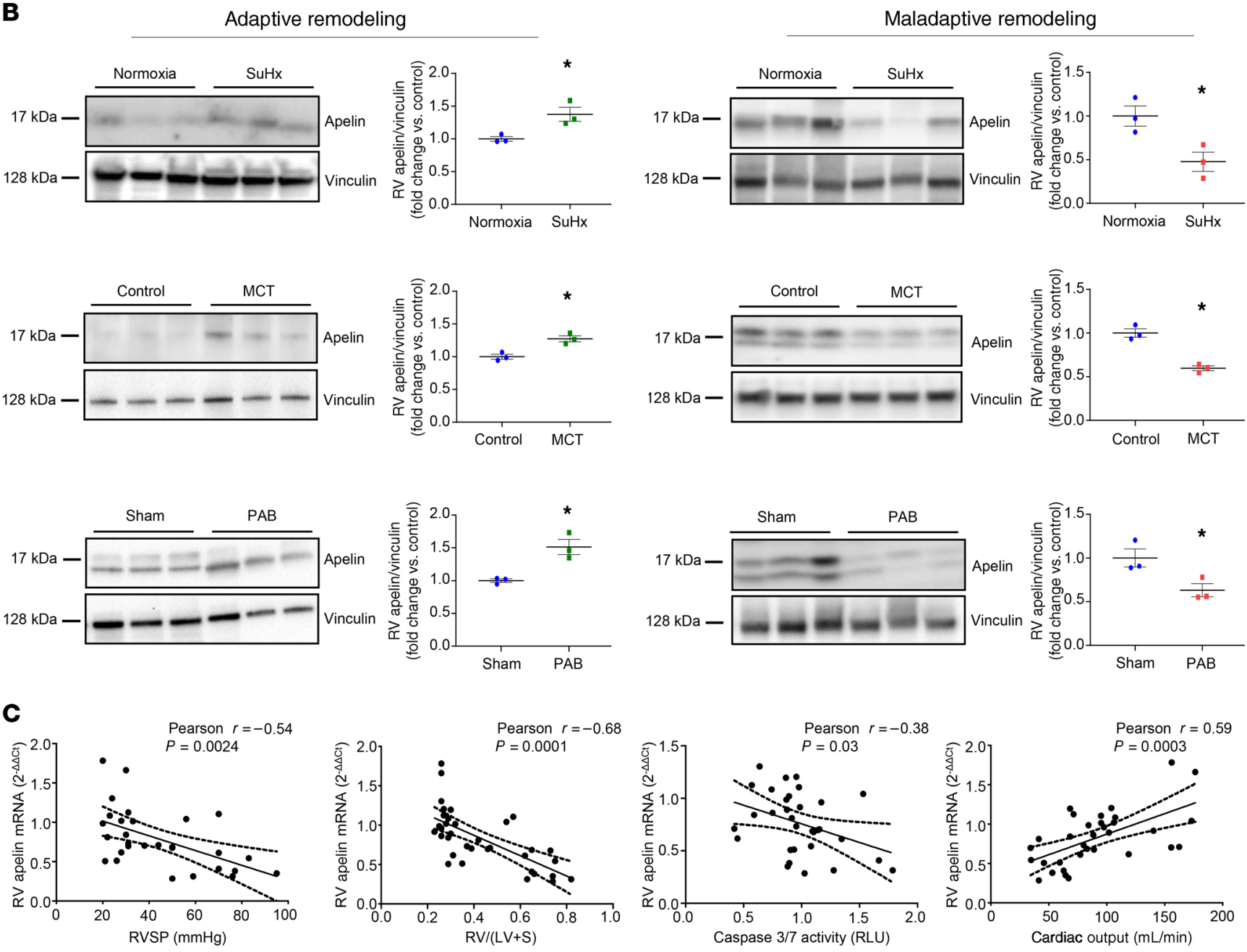

D
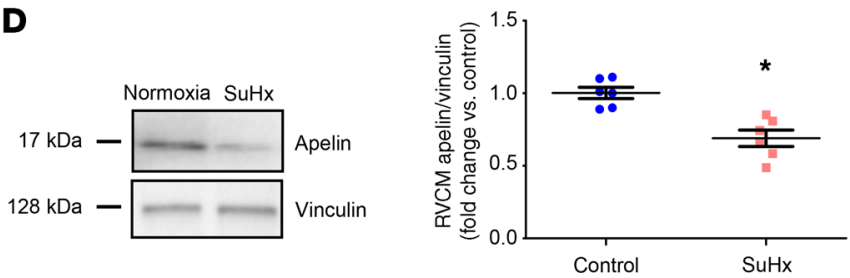

Figure 3. RV apelin expression is decreased in maladaptive (but not adaptive) RV hypertrophy and correlates negatively with markers of worsening RV function. (A) Apelin and APLNR are expressed in the RV. Apelin (middle image) and APLNR (right image) stained by immunohistochemistry in the RV of a male Sprague-Dawley rat. Both apelin and APLNR are expressed in coronary endothelial cells (arrows) as well as cardiomyocytes (arrowheads). Images are 10x; scale bars: $50 \mu \mathrm{m}$. (B) Apelin expression by Western blot and quantified via densitometric analyses in RVs from rats with adaptive remodeling (characterized by preserved cardiac output; left panel) or maladaptive remodeling (characterized by reduced cardiac output; right panel) employing rats with $\mathrm{SuHx}-\mathrm{PH}$, monocrotaline-induced PH (MCT), or pulmonary artery banding (PAB). Note decrease in apelin in maladaptive but not adaptive RV hypertrophy. $n=3$ male rats/group. (C) Apelin mRNA correlates negatively with RV systolic pressure (RVSP), RV hypertrophy [RV weight divided by weight of left ventricle + septum; RV/(LV + S)], and proapoptotic signaling (caspase-3/7 activity; in relative light units [RLU]), but positively with cardiac output in male and female control rats and rats with SuHx-PH (hemodynamics described in ref. 14). (D) Apelin expression in RV cardiomyocytes (RVCMs) isolated from normoxia control or SuHx-PH male and female rats. A representative Western blot is shown on the left; densitometry is shown on the right. ${ }^{*} P<0.05$ by Student's $t$ test in $\mathbf{B}$ and $\mathbf{D}$. Error bars in $\mathbf{B}$ and $\mathbf{D}$ represent mean \pm SEM; each data point represents 1 animal. Correlation analyses in $\mathbf{C}$ performed by determining Pearson's correlation coefficient $(R)$ and 2-tailed $P$ value. Dashed lines represent $95 \% \mathrm{CI}$. 
A

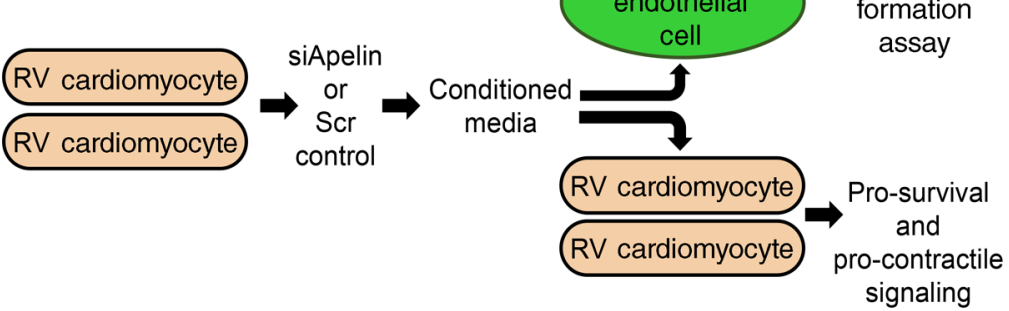

C

D

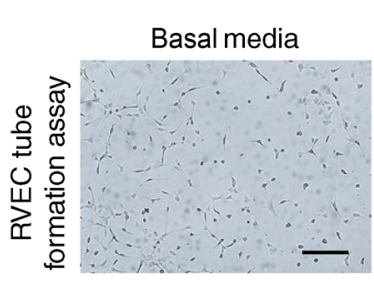

siApelin

RV cardiomyocyte conditioned media

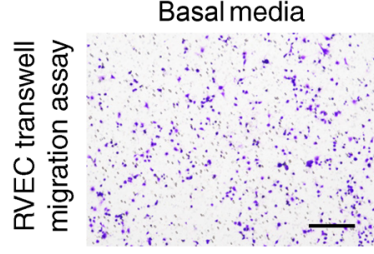
conditioned media
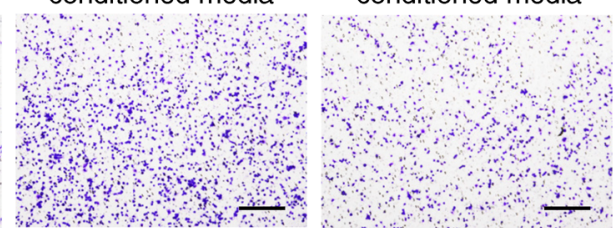

Scr control RV cardiomyocyte conditioned media

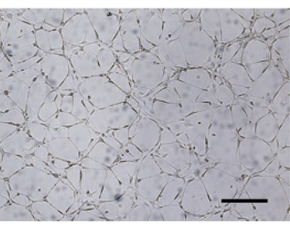

siApelin RV cardiomyocyte conditioned media
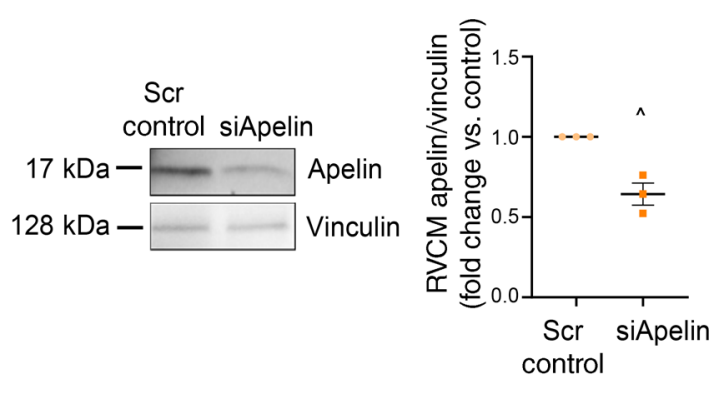

B

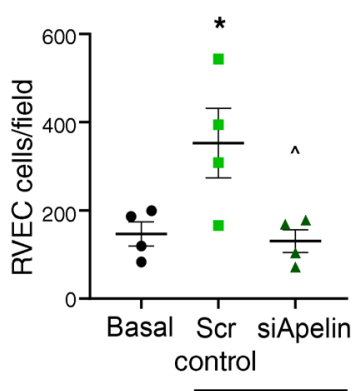

RVCM

cond. med.

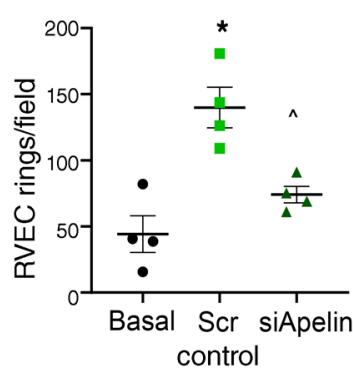

RVCM

cond. med.

E

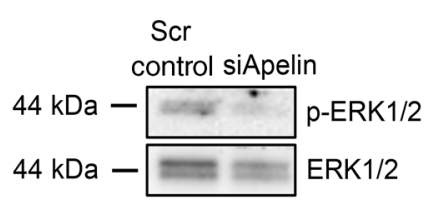

$\mathbf{F}$

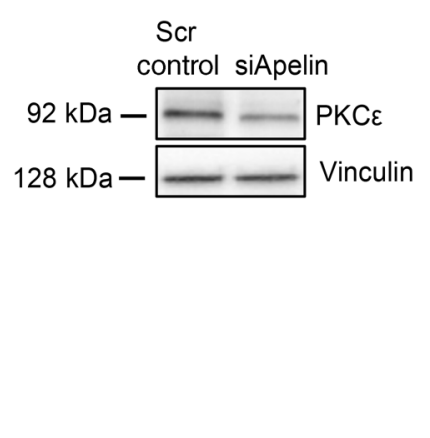

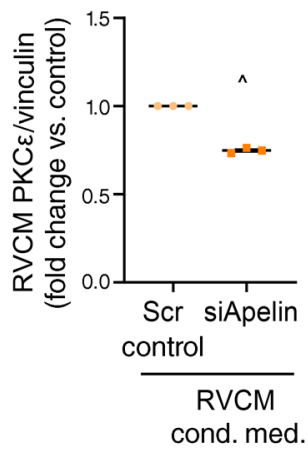


Figure 4. RV cardiomyocyte apelin exerts paracrine effects on RV endothelial cell and RVCM function. (A) RVCMs were transfected with siRNA directed against apelin (siApelin) or scrambled control (Scr control). 24 hours later, conditioned media was collected from RVCMs and added to naive RVECs or RVCMs. RVEC function and RVCM mediators of prosurvival and procontractile signaling were then assessed. (B) Validation of apelin siRNA knockdown in RVCMs. Scrambled siRNA oligo served as control. $n$ = 3 male rats. (C) Transwell migration assay in RVECs treated with RVCM conditioned media after treatment with siApelin or scrambled control. EBM2 media served as baseline control. Fifteen fields per condition were quantified 16 hours after conditioned media was added. Representative Transwell migration assay images are shown, with quantification on the right. Images are at 10× magnification, scale bars: $250 \mu \mathrm{m}$. $n=$ RVECs from 4 male rats, performed in technical triplicate. (D) Tube formation assay in RVECs treated with RVCM conditioned media after treatment with siApelin or scrambled control. EBM2 media served as baseline control. Cells plated at a density of $5 \times 10^{4}$ in technical triplicate. Images taken at $10 \times$ magnification; scale bars: $250 \mu \mathrm{m}$. Rings quantified using 15 fields per condition. Representative images are shown, with quantification of ring formation depicted on the right. $n=$ RVECs from 4 male rats (in technical triplicate). (E and $\mathbf{F}$ ) Quantification of ERK1/2 activation and PKC- $\varepsilon$ expression in RVCMs treated with RVCM conditioned media after treatment with siApelin or scrambled control. Representative Western blot images are shown, with quantification by densitometry depicted on the right. $n=$ RVCMs from 3 male rats. ${ }^{*} P<0.05$ versus basal media control, ${ }^{\wedge} P<0.05$ versus Scr control by (C and D) 1-way ANOVA with post hoc Tukey's correction or ( $\mathbf{E}$ and $\mathbf{F}$ ) by Student's $t$ test. Error bars represent mean \pm SEM; each data point represents cells from 1 animal.

respectively (Figure 5, E and F). These data identified E2 as a potent inducer of apelin in RVCMs.

Apelin signaling is necessary for E2-mediated stimulation of prosurvival signaling in vitro and for $E 2$ to exert $R V$-protective effects in vivo. We next studied whether E2 stimulates survival signaling in the RV, and whether apelin is necessary for $\mathrm{E} 2$ to exert this effect. In RVCMs isolated from male SuHx-PH rats, activation of ERK1/2 was decreased, whereas no such decrease was noted in RVCMs from E2-treated SuHx-PH male rats (Supplemental Figure 5A). Similarly, in H9c2 cells, pretreatment with E2 prevented TNF- $\alpha-$ induced decreases in ERK1/2 activation (Supplemental Figure 5B). Knockdown studies demonstrated that E2 increased prosurvival signaling in cardiomyocytes in an apelin-dependent manner (Supplemental Figure 5B).

To study whether apelin signaling is necessary for E2 to exert $\mathrm{RV}$ protection in vivo, we treated male $\mathrm{PAB}$ rats with $\mathrm{E} 2$ with or without APLNR antagonist ML221. Indeed, ML221 cotreatment resulted in loss of protective effects of E2 on RV hypertrophy, cardiac output, stroke volume, and neurohormonal activation (Supplemental Figure 6A-E). Similarly, ML221 cotreatment resulted in decreased RV ERK1/2 activation and PKC- $\varepsilon$ expression compared with E2 alone (Supplemental Figure 6F). These data indicate that apelin and APLNR signaling are necessary for E2 to exert RV protection.

$E R-\alpha$ is necessary and sufficient to increase apelin. Previous studies in SuHx-PH rats identified correlations between RV ER- $\alpha$ expression and apelin mRNA abundance as well as RV structure and function (12). We expanded these findings by demonstrating a robust correlation between ER- $\alpha$ and apelin protein expression in SuHx-PH RVs (Figure 6A). To evaluate whether this ER- $\alpha$ /apelin relationship extends to a large animal model, we studied correla- tions in RVs from yearling steers with and without high altitudeinduced PH. We found robust correlations between ER- $\alpha$ and apelin mRNA (Supplemental Figure 7, A and D). In conjunction with the data demonstrating a correlation between ER- $\alpha$ and apelin protein in human RVs (Figure 2B), these data indicate an ER- $\alpha$ / apelin relationship that extends across several species. ER- $\beta$, on the other hand, did not correlate with apelin or RV function in human RVs (Supplemental Figure 1, B and C) or rat RVs (Supplemental Figure 8).

Next, we investigated whether ER- $\alpha$ is necessary for E2mediated upregulation of apelin. Using siRNA, we identified that $\mathrm{ER}-\alpha$ was indeed necessary for E2 to upregulate apelin in $\mathrm{H} 9 \mathrm{c} 2$ cells (Figure 6B). We then treated H9c2 cells with the ER- $\alpha$-selective agonist BTP- $\alpha$ (28) and determined that ER- $\alpha$ activation was sufficient to induce apelin expression (Figure 6C). This effect extended to RVCMs from healthy male rats, where ex vivo treatment with E2 or BTP- $\alpha$ more than doubled apelin abundance (Figure 6D). Similarly, BTP- $\alpha$ increased apelin abundance in RVCMs from male and female SuHx-PH rats, albeit to a slightly lesser degree than in RVCMs from healthy rats (Figure 6E). ER- $\alpha$ activation was also sufficient to increase apelin in vivo, evidenced by a $30 \%$ increase in RV apelin mRNA expression in male SuHx-PH rats treated with ER- $\alpha$ agonist 4,4',4' -(4-propyl-[1H]pyrazole-1,3,5-triyl)trisphenol (PPT) that recapitulated increases seen with E2 treatment (Figure 6F). This increase occurred at the cardiomyocyte level, in that RVCMs isolated from $\mathrm{SuHx}-\mathrm{PH}$ male rats treated with E2 or PPT exhibited increased apelin abundance (Figure 6G). Two in vivo loss-of-function studies solidified these data: First, in RVs from chronically hypoxic E2-treated WT, ER- $\alpha$ KO $\left(E s r 1^{-/-}\right)$, or ER- $\beta-$ KO (Esr $\left.2^{-/}\right)$male mice, ER- $\alpha$ (but not ER- $\beta$ ) was necessary for E2 to increase apelin (Figure 7A). Second, RV apelin expression was decreased in ER- $\alpha$ loss-of-function mutant male and female rats exposed to hypoxia (Figure 7B). ER- $\alpha$ mutant rats also exhibited a decrease in cardiac index (versus WT; Figure $7 C)$, suggesting that ER- $\alpha$ exerts stimulatory effects on RV function. Together, these data suggest that E2 increases RVCM apelin via ER- $\alpha$ in vitro and in vivo.

ER- $\alpha$ is necessary for E2 to attenuate cardiopulmonary dysfunction in severe $P H$. Next, we determined whether ER- $\alpha$, in addition to being necessary for E2 to increase apelin, is also necessary for E2 to improve cardiopulmonary hemodynamics. We treated male or ovariectomized female WT or ER- $\alpha$ loss-of-function mutant SuHx-PH rats with E2. As shown previously, we found that E2 prevented $\mathrm{PH}$-induced cardiopulmonary dysfunction (Figure 8, A-E). However, this was not observed in ER- $\alpha$ mutant rats, suggesting that ER- $\alpha$ indeed is necessary for E2 to exert protective effects in the cardiopulmonary system (Figure 8, A-E). As in our in vitro and mouse studies, ER- $\alpha$ was indispensable for E2 to increase RV apelin (Figure 8F).

E2 increases BMPR2 in RVCMs. Since BMPR2 increases apelin in the lung vasculature (29), and since the BMPR2 promoter has an estrogen response element (30), we studied whether BMPR2 is a target of E2 in the RV. BMPR2 was indeed expressed in the RV, and its abundance inversely correlated with SuHxinduced increases in RVSP $(r=-0.66, P=0.01)$ and RV mass $(r$ $=-0.65, P=0.01)$. Important to our hypothesis, in $\mathrm{H} 9 \mathrm{c} 2$ cells, E2 attenuated staurosporine- or TNF- $\alpha$-induced decreases 
A

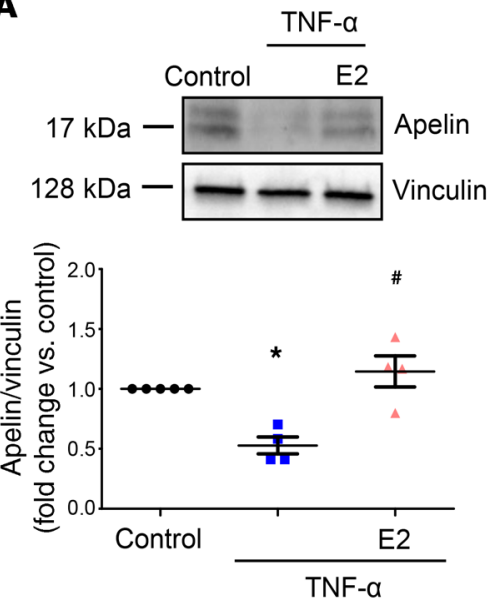

D

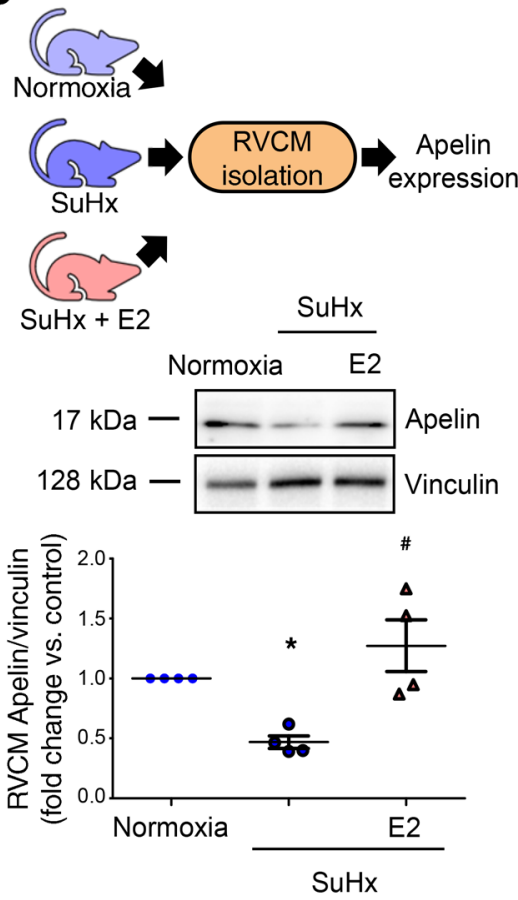

B
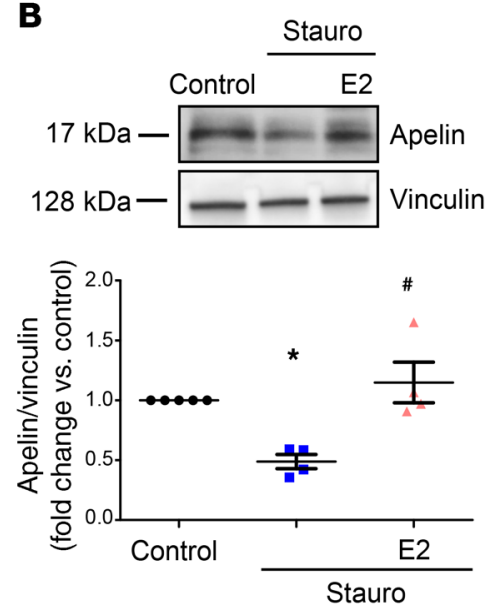

E
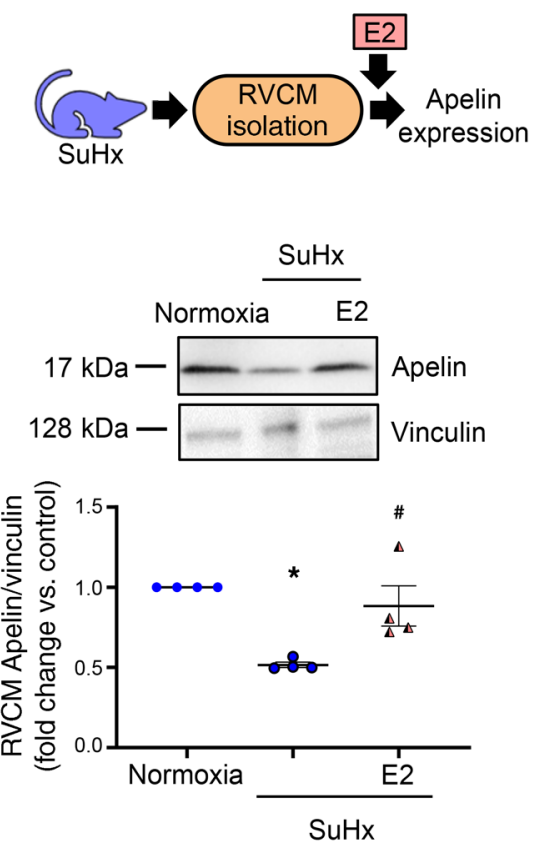

C
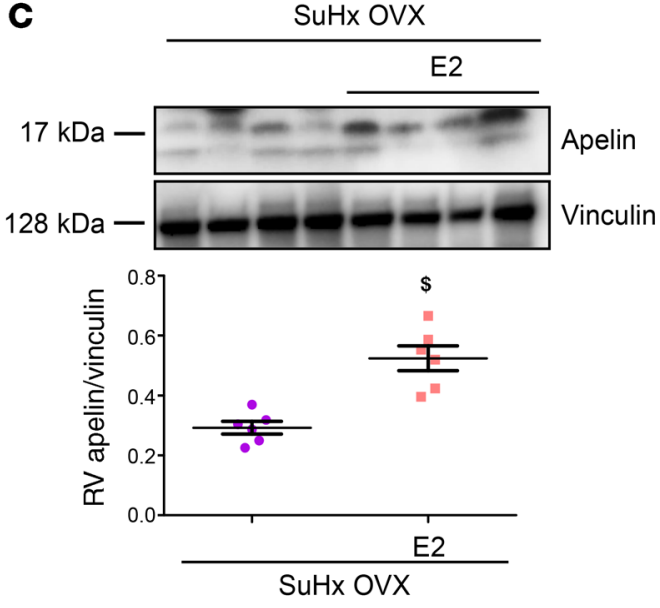

$\mathbf{F}$

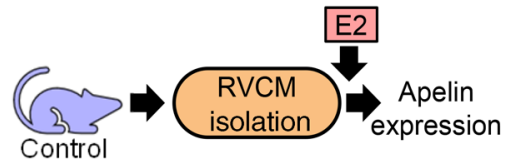

Figure 5. 17ק-estradiol increases apelin expression in stressed cardiomyoblasts, in the failing RV, and in isolated RV cardiomyocytes. (A and B) H9c2 rat cardiomyoblasts were pretreated with E2 (100 nM, 24 hours) and then stressed with TNF- $\alpha(10 \mathrm{ng} / \mathrm{ml}$, 8 hours; A) or staurosporine (stauro; $50 \mathrm{nM}, 4$ hours; B). (C) RVs from female SuHx OVX (ovariectomized SuHx-PH females) or SuHx OVX + E2 (ovariectomized SuHx-PH females replete with E2 $75 \mu \mathrm{g} / \mathrm{kg} /$ day via s.c. pellets) analyzed for apelin expression by Western blot. (D) Apelin expression evaluated by Western blot in RVCMs isolated from male normoxia control, untreated SuHx-PH, and SuHx-PH rats treated with E2 (75 $\mu \mathrm{g} / \mathrm{kg} /$ day; s.c. pellets) in vivo. (E) Western blot analysis of apelin expression in RVCMs isolated from male and female SuHx-PH rats and treated exogenously with E2 (10 nM, 24 hours). (F) Western blot analysis of apelin expression in RVCMs isolated from control male rats treated with E2 (10 nM, 24 hours) in vitro. $n=4$ independent experiments in $\mathbf{A}$ and $\mathbf{B}, n=6$ rats/group in C, cardiomyocytes isolated from $n=4-5$ rats/group in D-F, with each data point indicating 1 animal. All panels demonstrate representative Western blots with densitometric analyses for all experiments or animals. ${ }^{*} P<0.05$ versus control/normoxia; ${ }^{*} P<0.05$ versus TNF-treated (A), stauro-treated (B), or untreated SuHx (D and $\mathbf{E}) ;{ }^{\$} P<0.05$ versus OVX. $P$ values in $\mathbf{A}, \mathbf{B}, \mathbf{D}$, and $\mathbf{E}$ by ANOVA with Dunnett's post hoc correction; $P$ values in $\mathbf{C}$ and $\mathbf{F}$ by Student's $t$ test. Error bars represent mean \pm SEM; each data point represents 1 experiment or animal.

in BMPR2 (Figure 9, A and B). E2 also increased BMPR2 in RVCMs isolated from control male or SuHx-PH male and female rats (Figure 9, C and D). In vivo, E2 repletion restored SuHx-induced decreases in RV BMPR2 expression from male and female rats (Figure 9E). PH-induced decreases in BMPR2 in RVCMs isolated from SuHx-PH male rats were prevented with in vivo E2 treatment (Figure 9F). These data suggest that $\mathrm{E} 2$ increases BMPR2 in RVCMs in vitro and in vivo.
$E R-\alpha$ is necessary and sufficient to increase BMPR2. We next determined the role of ER- $\alpha$ in E2-mediated increases in BMPR2. In SuHx-PH RVs from male and female rats, ER- $\alpha$ protein correlated positively with BMPR2 abundance (Figure 10A). Similarly, a correlation between ER- $\alpha$ and BMPR 2 mRNA was found in RVs from yearling steers (Supplemental Figure 7, B and E).

We then evaluated whether ER- $\alpha$ binds to the Bmpr 2 promoter in the presence of E2 by performing a ChIP assay in E2-treated 
A

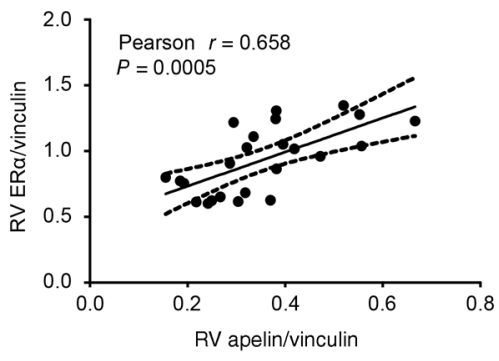

C

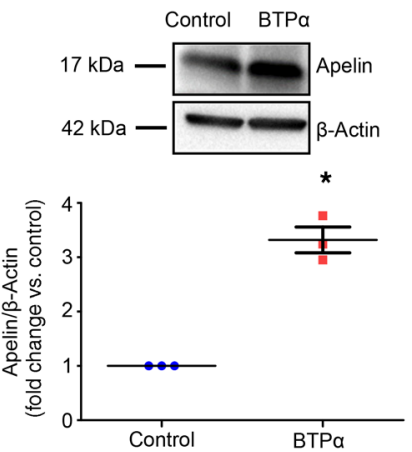

$\mathbf{F}$

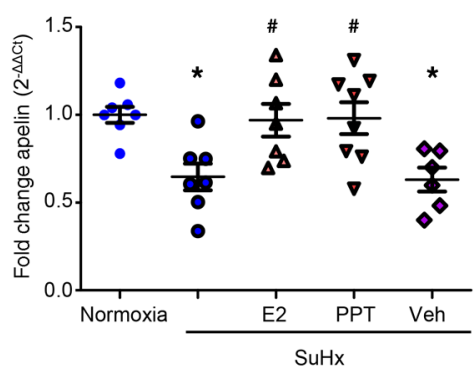

B
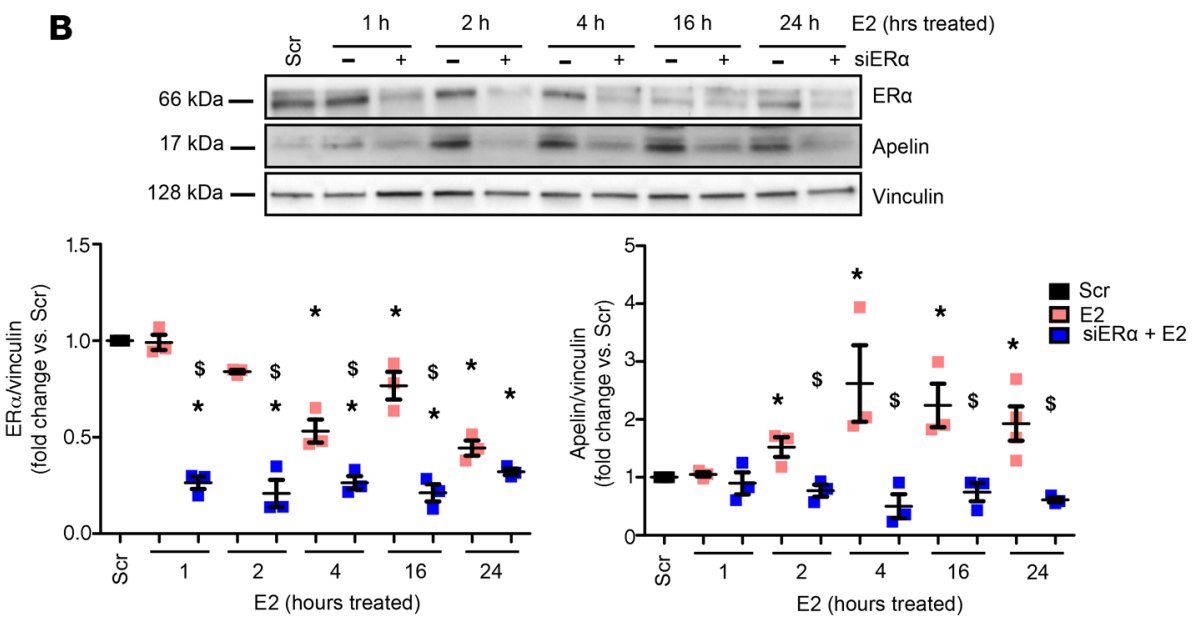

D

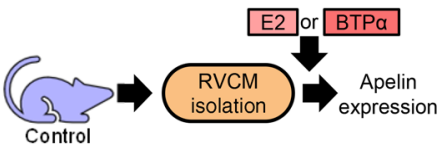

E
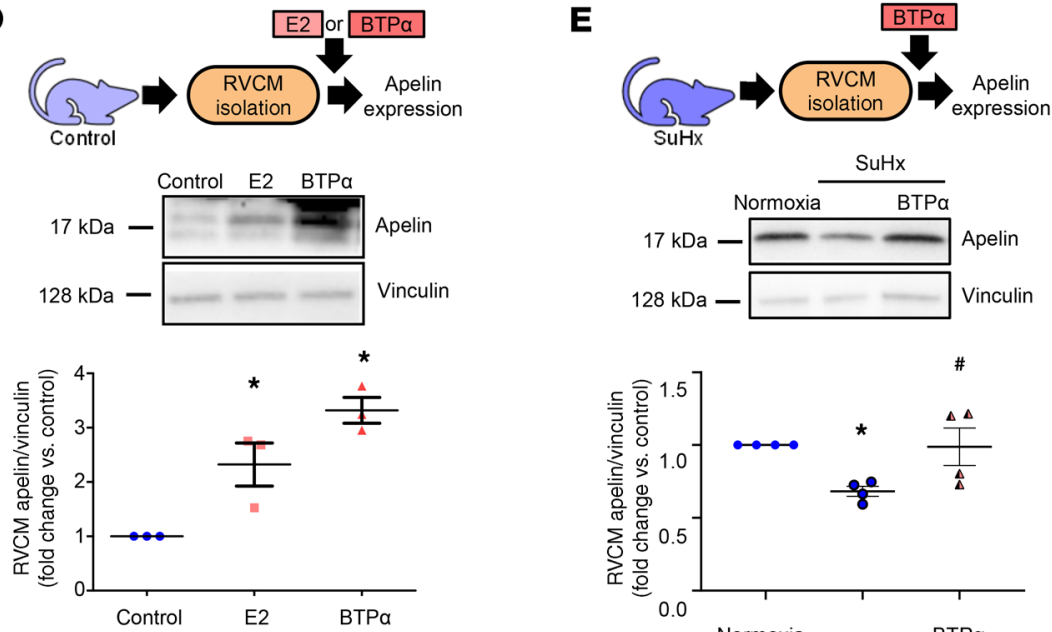

G

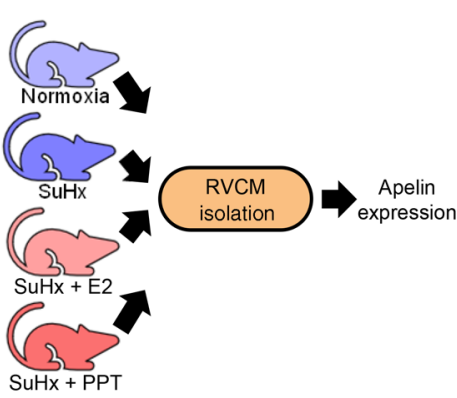

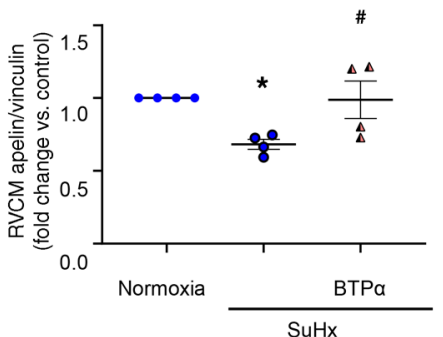
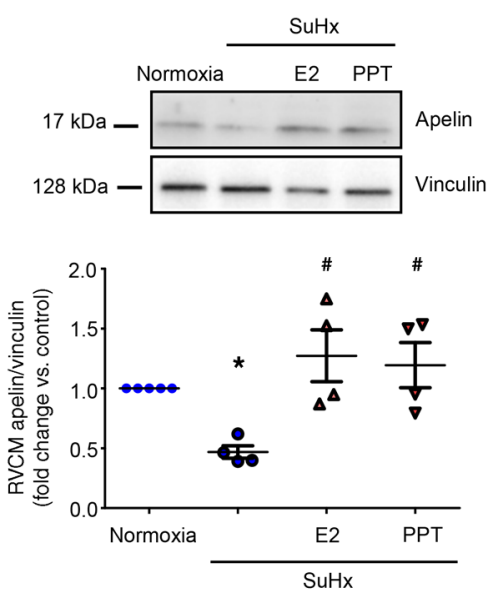

Figure 6. ER- $\alpha$ is necessary and sufficient for upregulating apelin in vitro. (A) RV apelin and ER- $\alpha$ protein positively correlate in male and female control and SuHx-PH rats. (B) ER- $\alpha$ siRNA knockdown time course in H9c2 cardiomyoblasts (5 nM; 24 hours prior to E2 [100 nM]). (C) Apelin expression in H9c2 cells treated with ER- $\alpha$ agonist BTP- $\alpha$ (100 nM, 24 hours). (D) Apelin expression in RV cardiomyocytes (RVCMs) isolated from male control rats treated with E2 (10 nM, 24 hours) or BTP- $\alpha$ (100 nM, 24 hours) in vitro. (E) Apelin expression in RVCMs isolated from male and female SuHx-PH rats and treated with BTP (100 nM, 24 hours) in vitro. (F) RV apelin mRNA expression in normoxia, SuHx-PH, or SuHx-PH rats treated with E2 (75 $\mu \mathrm{g} / \mathrm{kg} / \mathrm{day}$ via s.c. pellets), ER- $\alpha$ agonist PPT ( $850 \mu \mathrm{g} / \mathrm{kg} /$ day; s.c. pellets) or EtOH vehicle (Veh). (G) Apelin expression in RVCMs isolated from male controls, SuHx-PH, or SuHx-PH rats treated with E2 or PPT in vivo. $n=3$ independent experiments for $\mathbf{B}$ and $\mathbf{C}$. Cells from $n=3-4$ rats/group in $\mathbf{D}, \mathbf{E}$, and $\mathbf{G}$. $n=6-8 /$ group in $\mathbf{F}$. B-E and $\mathbf{G}$ depict representative Western blots with densitometric analyses. Pearson's $R$ value and $P$ value shown in $\mathbf{A}$. Dashed lines represent $95 \% \mathrm{Cl}$. ${ }^{*} P<0.05$ versus Scr (scrambled control), ${ }^{\$} P<0.05$ versus E2 in $\mathbf{B} ;{ }^{*} P<0.05$ versus control in $\mathbf{C}$ and $\mathbf{D} ;{ }^{*} P<0.05$ versus Normoxia, $\# P<0.05$ versus SuHx in $\mathbf{E}-\mathbf{C}$. $P$ values by ANOVA with Tukey's post hoc correction in B, D-G and by Student's $t$ test in $\mathbf{C}$. Error bars represent mean \pm SEM; each data point represents 1 experiment or animal. 
A
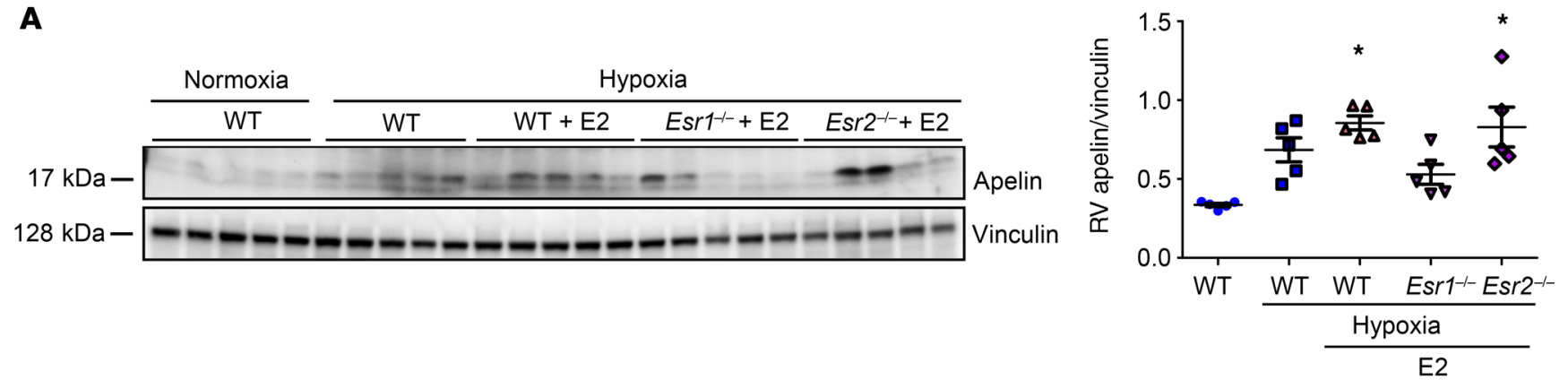

B
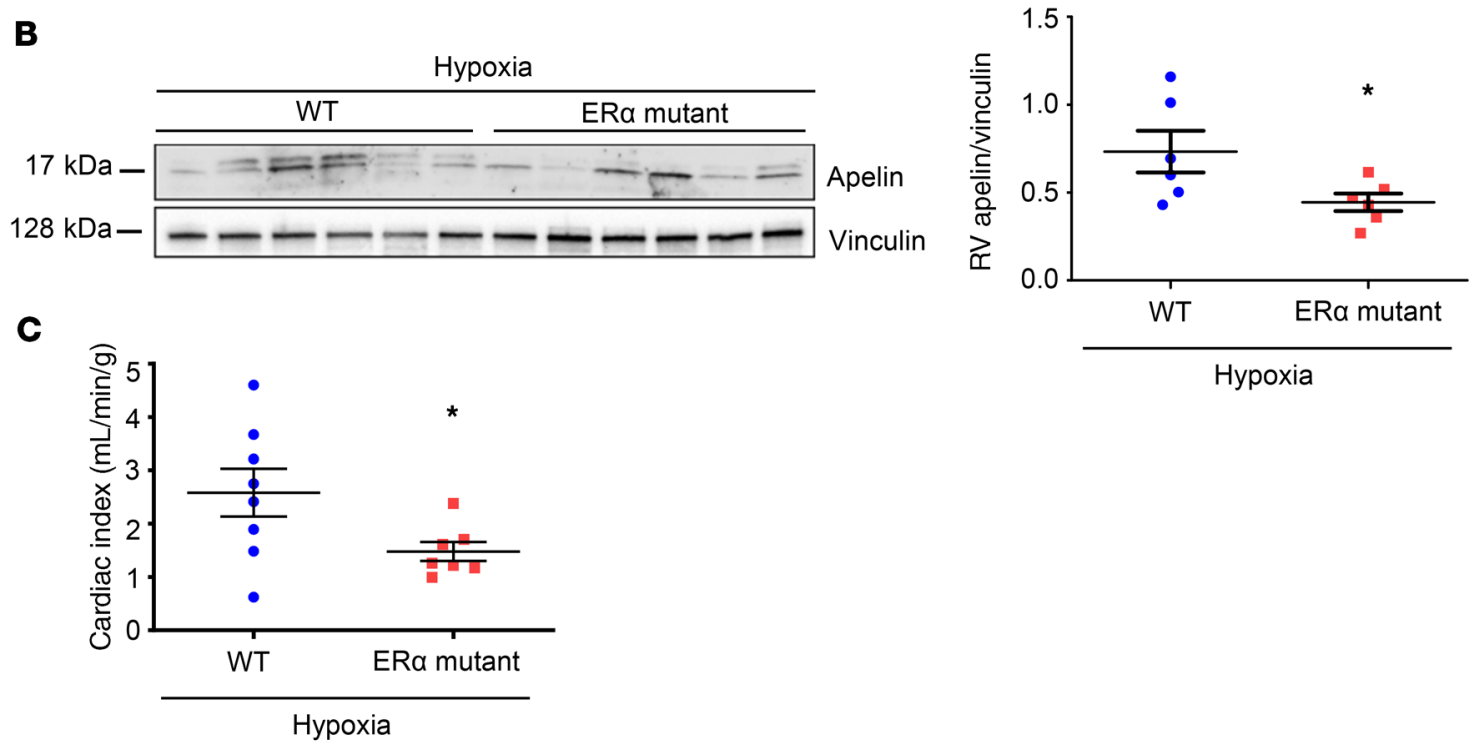

Hypoxia

Figure 7. ER- $\alpha$ is necessary for upregulating apelin in vivo. (A) RV apelin expression in male WT, ER- $\alpha-K O$ (Esr1), and ER- $\beta-K O$ (Esr2) mice with hypoxia-induced PH (HPH) treated with E2 (75 $\mu \mathrm{g} / \mathrm{kg} /$ day; s.c. pellets). (B) RV apelin expression and (C) cardiac indices (echocardiographic cardiac output/body weight) in male and female control and ER- $\alpha$ (Esr1) mutant HPH rats. $n=5 /$ group in $\mathbf{A}, n=6 /$ group in $\mathbf{B}$ and $\mathbf{C}$. ${ }^{*} P<0.05$ versus WT. $P$ values by ANOVA with Tukey's post hoc correction in $\mathbf{A}$ and by Student's $t$ test in $\mathbf{B}$ and $\mathbf{C}$. Error bars represent mean \pm SEM; each data point represents 1 experiment or animal.

H9c2 cells. We detected time-dependent ER- $\alpha$ binding to the Bmpr2 promoter (Figure 10B). Similar to the effect on apelin, we found that in E2-treated H9c2 cells, BMPR2 upregulation was significantly blunted after ER- $\alpha$ knockdown (Figure 10C). Next, we established that treatment with ER- $\alpha$ agonist BTP- $\alpha$ was sufficient to increase BMPR2 independent of E2 in H9c2 cells (Figure 10D) and RVCMs from control male or SuHx-PH male and female rats (Figure 10, E and F). Furthermore, ER- $\alpha$ activation also increased expression of the BMPR2 targets p-Smad 1/5/9 and Id1 (Supplemental Figure 11A), suggesting that ER- $\alpha$ activation was sufficient to increase canonical BMPR2 signaling. This increase was similar to the increase noted with E2 (Supplemental Figure 11B). In vivo, treatment of male SuHx-PH rats with ER- $\alpha$ agonist PPT replicated E2's effects and induced a significant increase in RV BMPR2 (Figure 10G). Finally, RVCMs isolated from male SuHx-PH rats treated with E2 or PPT in vivo demonstrated abrogated SuHx-induced decreases in BMPR2 (Figure 10H). Taken together, these data demonstrated that ER- $\alpha$ bound to the Bmpr2 promoter, upregulated RVCM BMPR2 expression in vitro and in vivo, and was necessary and sufficient to increase BMPR2. These data also indicate that ER- $\alpha$ increased canonical as well as noncanonical BMPR2 downstream signaling.
BMPR2 is necessary for $E 2$ to upregulate apelin and exert cardioprotective effects. In pulmonary artery endothelial cells, BMPR2 promotes formation of a $\beta$-catenin/PPAR $\gamma$ complex that binds to the apelin promoter (30). To evaluate whether E2 stimulates this pathway in cardiac cells, we treated H9c2 cells with E2 and, using co-IP, indeed detected $\beta$-catenin/PPAR $\gamma$ complex formation (Figure 11A). We then determined whether BMPR2 is necessary for the E2-mediated upregulation of apelin. Indeed, BMPR2 knockdown in E2-treated H9c2 cells abrogated the E2-mediated upregulation of apelin (Figure 11B). ER- $\alpha$ expression was not affected by BMPR2 knockdown, confirming that ER- $\alpha$ is upstream of BMPR2 (Figure 11B). We next measured apelin abundance in $\mathrm{H} 9 \mathrm{c} 2$ cells stressed with TNF- $\alpha$ or staurosporine and treated with $\mathrm{E} 2$ with or without siRNA directed against BMPR2. E2 was unable to maintain apelin expression after BMPR2 knockdown, suggesting that BMPR2 was indeed necessary for E2 to increase apelin (Figure 11, C and D). Finally, in stressed cardiomyoblasts, BMPR2 knockdown blocked the E2-mediated upregulation of phospho-ERK1/2, a known downstream target of apelin (ref. 24 and Figure 11E). BMPR2-mediated regulation of apelin was further corroborated by a positive correlation between BMPR2 and apelin mRNA in RVs from year- 
A

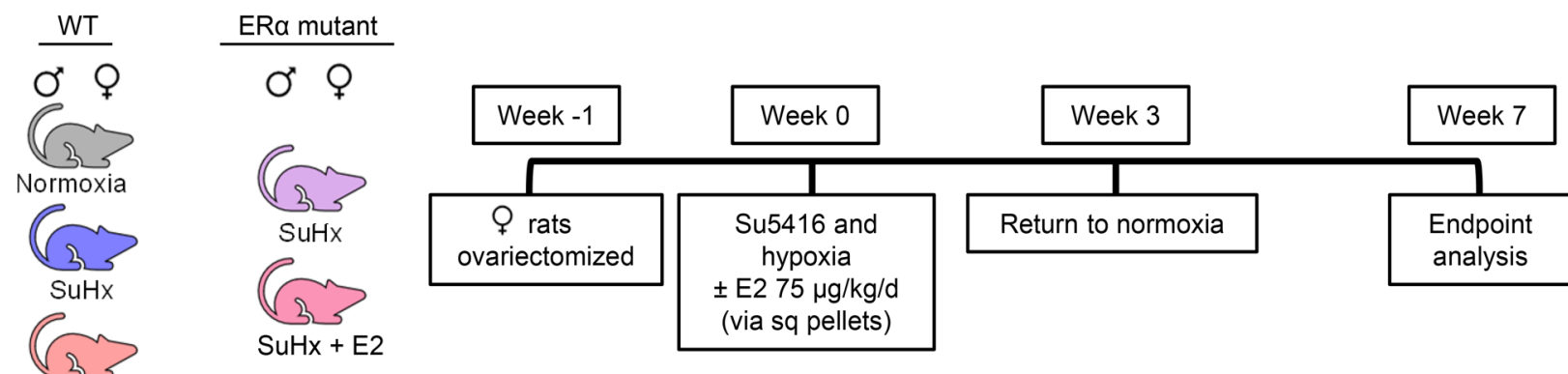

SuHx + E2

B

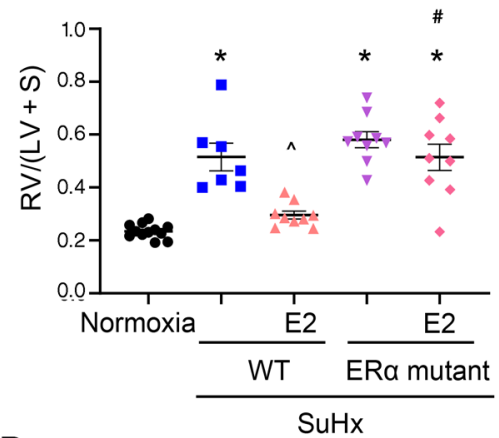

D

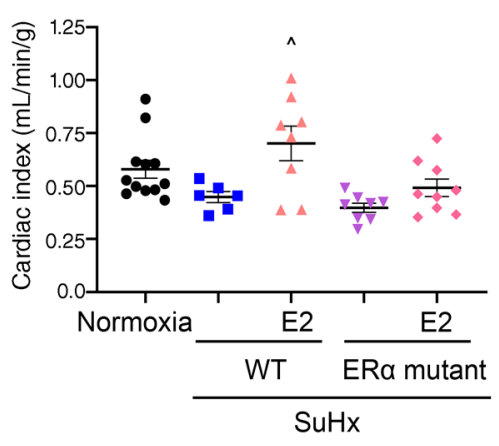

$\mathbf{F}$

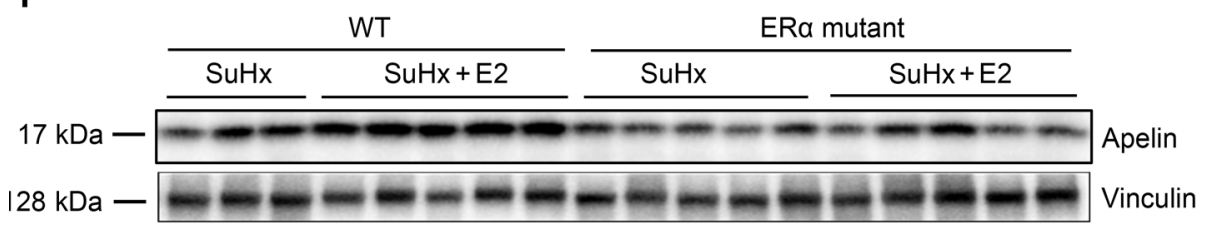

C

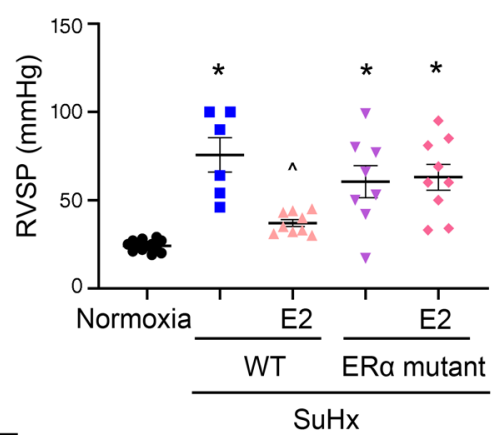

E

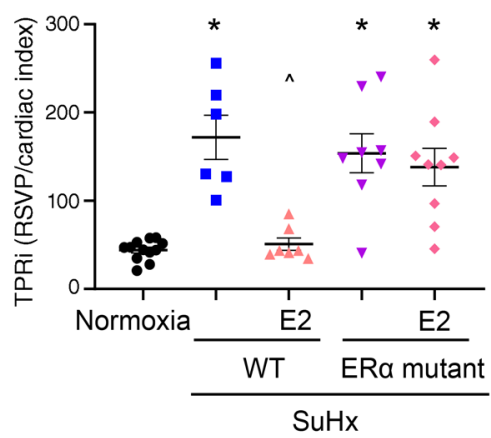

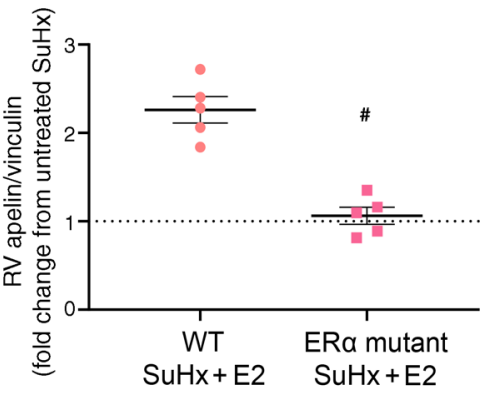

Figure 8. ER- $\alpha$ is necessary for E2 to attenuate cardiopulmonary dysfunction in SuHx-PH. (A) Experimental design. (B-E) Effects of E2 treatment in WT or ER- $\alpha$ loss-of-function mutants on RV hypertrophy (RV weight divided by weight of left ventricle plus septum; RV/[LV + S]; B), RV systolic pressure (RVSP; C), cardiac index (echocardiographic cardiac output/body weight; D), and total pulmonary resistance index (cardiac index/RVSP; E). (F) Western blot analysis of RV apelin. Densitometric analysis demonstrates decreased ability of E2 to mediate increase in RV apelin in ER- $\alpha$ loss-of-function mutant (data expressed as fold-change increase in $\mathrm{RV}$ apelin with $\mathrm{E2}$ versus untreated). ${ }^{*} P<0.05$ versus normoxia control, ${ }^{\wedge} P<0.05$ versus WT SuHx untreated, ${ }^{\#} P<$ 0.05 versus WT SuHx + E2 by 1-way ANOVA with Tukey post hoc correction in B-E. ${ }^{\#} P<0.05$ versus WT SuHx + E2 by $t$ test in $\mathbf{F}$. Error bars represent mean \pm SEM. Each data point represents 1 animal. Sq = subcutaneous.

ling steers (Supplemental Figure 7, C and F). These data suggest that $\mathrm{E} 2$ promoted formation of a $\beta$-catenin/PPAR $\gamma$ complex and indicate that BMPR2 was necessary for E2 to increase apelin expression and ERK1/2 phosphorylation. These findings, in con- junction with data presented in Figure 10, support the presence of an E2/ER- $\alpha$ /BMPR2/apelin axis in the RV.

To study whether BMPR2 is essential for E2 to increase apelin and attenuate RV dysfunction in vivo, we employed male or 
A

B

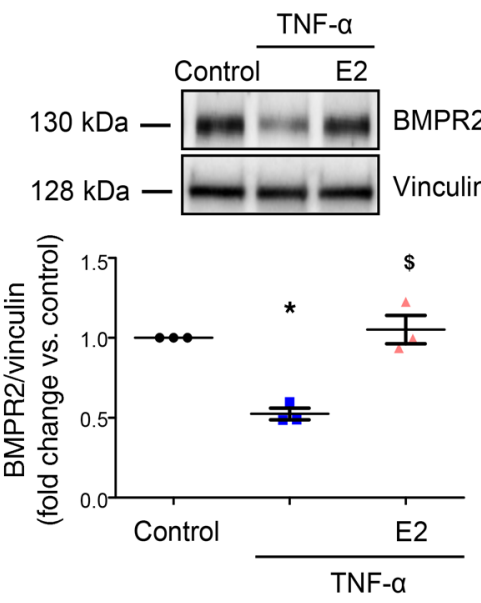

D

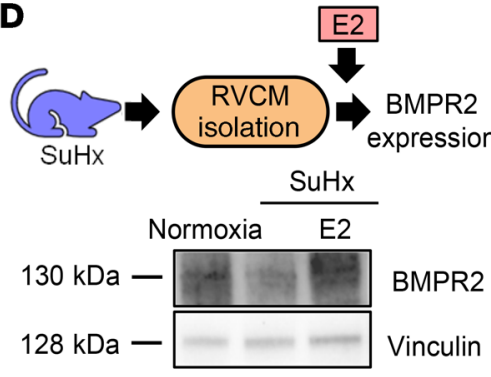

C

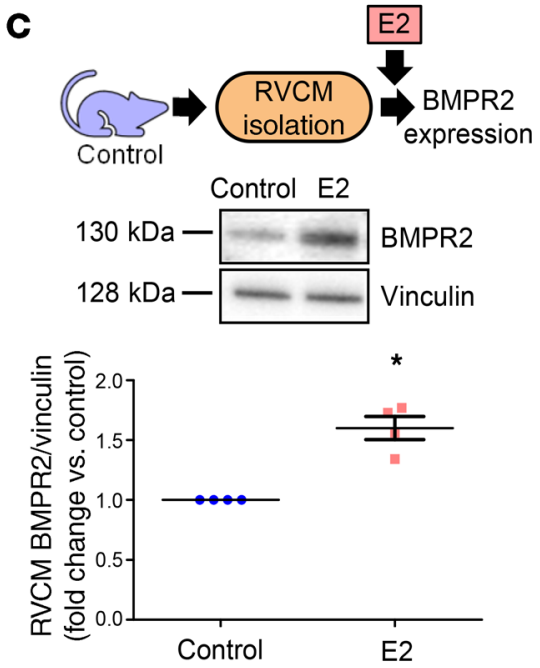

E
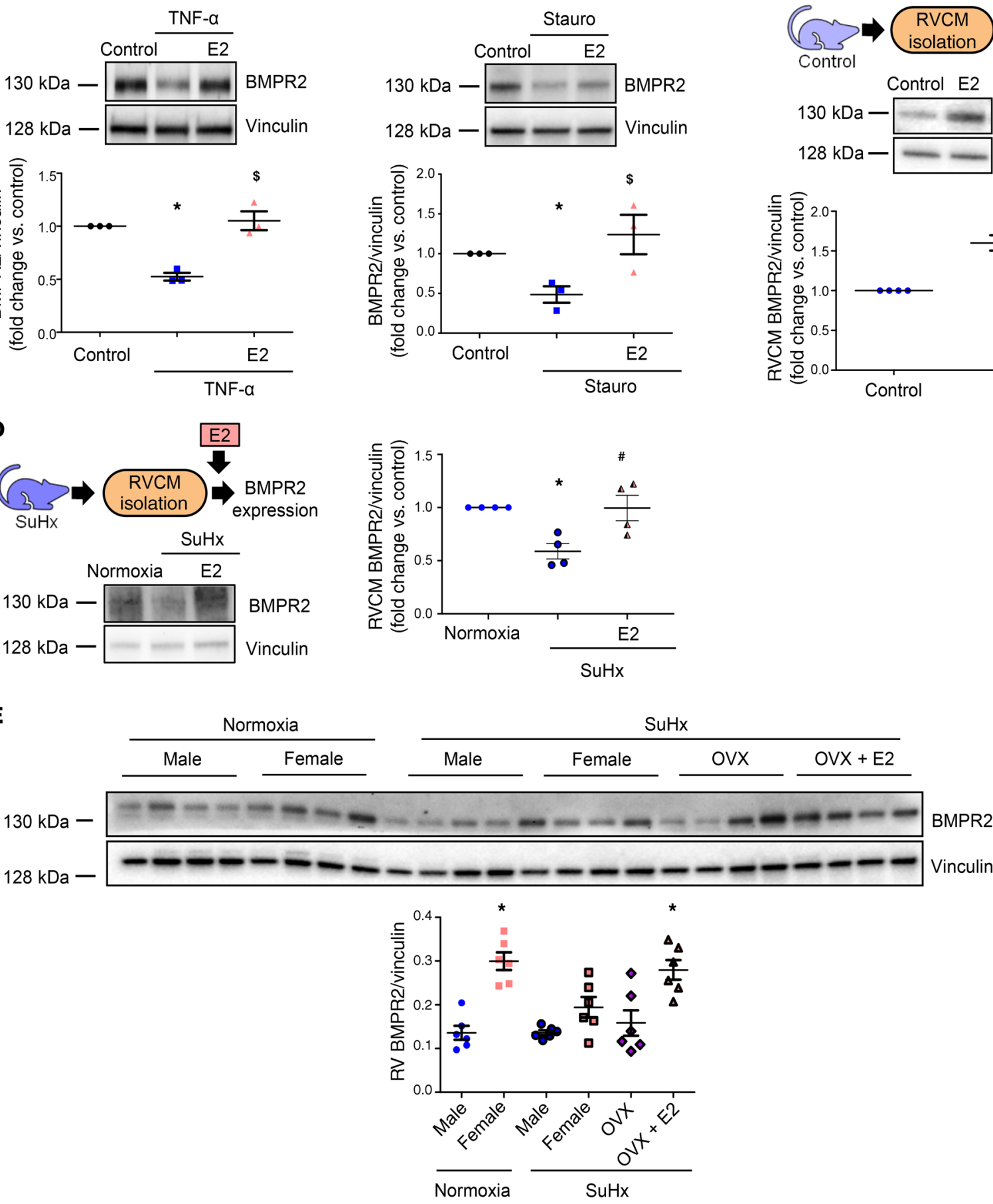

$\mathbf{F}$
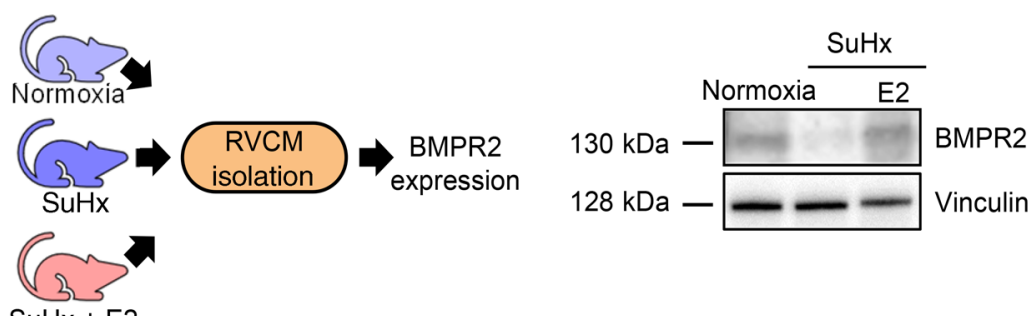

$\mathrm{SuHx}+\mathrm{E2}$

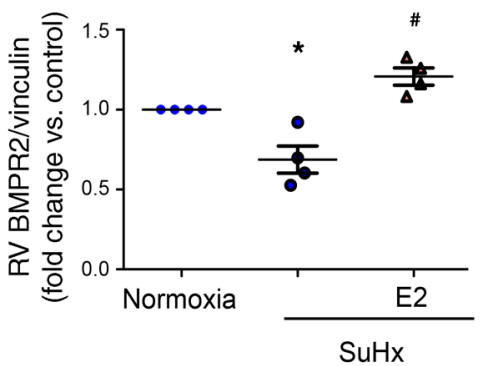


Figure 9. E2 upregulates BMPR2 in stressed cardiomyoblasts, in SuHx-PH $\mathbf{R V s}$, and in RV cardiomyocytes. (A and B) Effects of E2 on BMPR2 expression in H9c2 cardiomyoblasts treated with TNF- $\alpha(10 \mathrm{ng} / \mathrm{ml}$, 8 hours; (A) or staurosporine (stauro; $50 \mathrm{nM}, 4$ hours; (B). Cells were pretreated with E2 (100 nM) for 24 hours prior to TNF/stauro exposure and then lysed and analyzed by Western blot. (C) Western blot of BMPR2 expression in RVCMs isolated from male control rats treated with E2 in vitro ( $10 \mathrm{nM}, 24$ hours). (D) Western blot of BMPR2 expression in RVCMs isolated from male and female SuHx-PH rats and treated in vitro with E2 (10 nM, 24 hours). (E) Effects of endogenous or exogenous E2 on RV BMPR2 expression. SuHx$\mathrm{PH}$ was induced in male, intact female, and ovariectomized (OVX) female rats. A subgroup of OVX females was replete with E2 $(75 \mu \mathrm{g} / \mathrm{kg} /$ day via s.c. pellets for 7 weeks). Note higher baseline BMPR2 expression in female controls compared with males and increased RV BMPR2 protein abundance after E2 treatment. (F) BMPR2 expression evaluated by Western blot in RVCMs isolated from male normoxic control rats, untreated SuHx-PH rats, and SuHx-PH rats treated with E2 $(75 \mu \mathrm{g} / \mathrm{kg} /$ day; s.c. pellets) for 7 weeks. $n=3$ independent experiments in $\mathbf{A}$ and $\mathbf{B}$; cells isolated from $n=4$ rats/group in $\mathbf{C}, \mathbf{D}$, and $\mathbf{F}$, with each data point indicating 1 animal; $n=6$ rats/group in E. Figures depict representative Western blots with densitometric analyses for all experiments. ${ }^{*} P<0.05$ versus untreated control, ${ }^{\$} P<0.05$ versus TNF or stauro treatment in $\mathbf{A}$ and $\mathbf{B}$; ${ }^{*} P<0.05$ versus untreated control in $\mathbf{C} ;{ }^{*} P<0.05$ versus normoxia control, ${ }^{\#} P<0.05$ versus untreated SuHx in $\mathbf{D}$ and $\mathbf{F}$. ${ }^{*} P<0.05$ versus male normoxic control in $\mathbf{E}$. All $P$ values by ANOVA with post hoc Tukey correction except for $\mathbf{C}$, where $P$ value is by Student's $t$ test. Error bars represent mean \pm SEM; each data point represents 1 experiment or animal.

ovariectomized female rats with a monoallelic deletion of $71 \mathrm{bp}$ in exon 1 of the Bmpr2 gene ( 471 rats, ref. 31 ) and induced RV failure by $\mathrm{PAB}$. We treated subgroups of these rats with $\mathrm{E} 2$ and compared E2's effects to those in E2-treated PAB WT controls. E2 increased cardiac output in PAB WT but was unable to do this in $\Delta 71$ rats (Supplemental Figure 13A). Similarly, E2 lowered RV end-diastolic pressure in PAB WT, but was not capable of lowering this parameter in $\Delta 71$ rats (Supplemental Figure 13B). Lastly, E2-treated PAB $\triangle 71$ rats exhibited a significantly prolonged pulmonary artery acceleration time compared with E2-treated PAB WT rats (Supplemental Figure 13C). Although E2 increased RV apelin expression in WT 1.4-fold, E2 was unable to increase RV apelin in $\Delta 71$ rats (Supplemental Figure 13D). These data indicate that BMPR2 was necessary for E2 to attenuate RV dysfunction and increase RV apelin expression in vivo.

E2 or ER- $\alpha$ agonist increases RV BMPR 2 and apelin and maintains $R V$ adaptation in established $\mathrm{PH}$. To determine whether E2 or PPT reverse-established $\mathrm{PH}$ and to assess effects of E2 or PPT on the entire cardiopulmonary axis, we used a rescue approach in the MCT-PH model (Figure 12A). Male MCT-PH rats were treated with E2 or PPT starting 2 weeks after MCT injection. Both E2 and PPT rescued MCT-induced decreases in RV BMPR2 and apelin (Figure 12B). Importantly, E2 or PPT decreased RVSP by $45 \%$ (Figure $12 \mathrm{C}$ ) and RV hypertrophy by $45 \%$ and $22 \%$, respectively (Figure 12D). Both compounds, administered when RV adaptation was still preserved, attenuated MCT-induced decreases in the cardiac index, resulting in a $60 \%$ higher cardiac index than in untreated MCT rats (Figure 12, E and G). In fact, E2 or PPT almost normalized the total pulmonary resistance index (Figure 12F). These changes occurred without E2 or PPT attenuating pulmonary vascular remodeling (Supplemental Figure 14). These data indicate that E2 and PPT increased RV BMPR2 and apelin and maintained
RV adaptation even in established $\mathrm{PH}$, and that these changes cannot be explained by indirect RV effects from decreased lung vascular remodeling.

$E 2$ exerts direct $R V$-protective effects that are independent of effects on RV afterload. Protective effects of E2 on RV function could be due to indirect effects from a lower RV afterload. While our studies in isolated RVCMs and PAB rats (Supplemental Figures 6 and 13) demonstrated direct effects of $\mathrm{E} 2$ on the RV, we aimed to study E2's RV effects in vivo in more detail. We administered E2 to PAB male rats employing a prevention approach (E2 starting at $\mathrm{PAB}$ ) as well as a delayed (treatment) approach (E2 starting 4 weeks after PAB; Figure 13A). The prevention approach indeed attenuated PAB-induced increases in RV hypertrophy and RV enddiastolic diameter (Figure 13, B and C). Furthermore, preventative $\mathrm{E} 2$ increased RVSP/RV mass compared with untreated PAB (Figure 13D), suggesting a higher force per contractile unit (32). E2-treated rats also exhibited a 3-fold higher stroke volume index (SVI) and a 3-fold higher cardiac index than untreated rats (Figure $13, \mathrm{E}-\mathrm{H})$. These changes were associated with decreased neurohormonal activation (Figure 13, I and J) and preservation of RV apelin expression in E2-treated animals (Figure 13K). Although effects of the treatment E2 approach were not as robust as those of preventative E2, this strategy resulted in significant ( $\geq 50 \%$ ) decreases in RV hypertrophy, RV end-diastolic diameter, and neurohormonal activation (Figure 13, B, C, I, and J) and a doubling (though not statistically significant) of the SVI and cardiac index compared with untreated controls (Figure 13, E-H). These data demonstrated that E2 had direct and afterload-independent effects on RV function and RV apelin expression in vivo.

E2 or ER- $\alpha$ agonist prolong survival in experimental $P H$ and $R V F$. Lastly, we evaluated whether E2 or ER- $\alpha$ improve survival in experimental RVF. Indeed, E2 or PPT (via a prevention protocol) decreased mortality in SuHx-PH compared with untreated rats (Supplemental Figure 15A). Similarly, in PAB rats, preventative E2 and E2 given via a delayed/treatment approach decreased mortality (Supplemental Figure 15B). These data suggest that RVprotective effects of E2 and ER- $\alpha$ in the cardiopulmonary axis translate into a robust survival benefit.

\section{Discussion}

Our studies identified a cardioprotective E2/ER- $\alpha$ /BMPR2/apelin axis in the RV (Supplemental Figure 15C). We describe a mechanism by which E2, via ER- $\alpha$, activates BMPR2 signaling to upregulate apelin, a potent effector of cardiac contractility (16). Although apelin is known to exert positive effects on RV function $(14,33)$, its regulation in the RV is not yet known. Similarly, the role and regulation of BMPR2 in the RV are incompletely understood. While BMPR2 and apelin are required for cardiac development and pulmonary vascular homeostasis (17-19), we now provide evidence that these mediators, employed via ER- $\alpha$, are active in the RV, providing a molecular basis for E2's RV-protective effects.

We previously demonstrated that E2 improves cardiac output, decreases RV mass, and favorably affects proapoptotic signaling, proinflammatory cytokine activation, and mitochondrial dysfunction in experimental RVF (12). We also showed that RV ER- $\alpha$ expression in healthy females is higher compared to males, tends to decrease with RVF in females and after OVX, increases with E2 
A

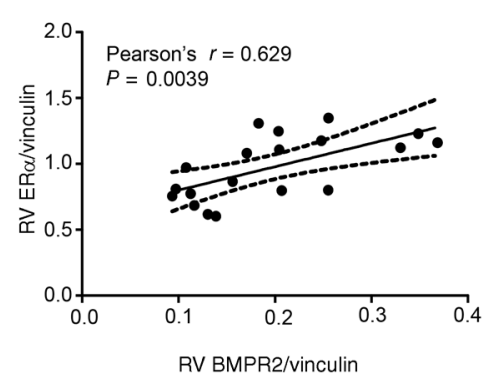

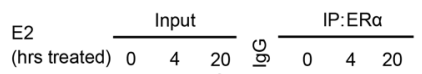
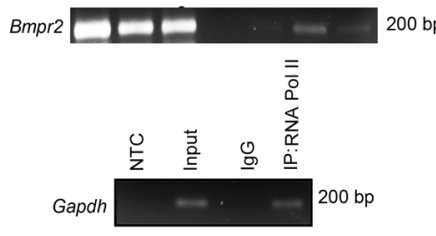

C
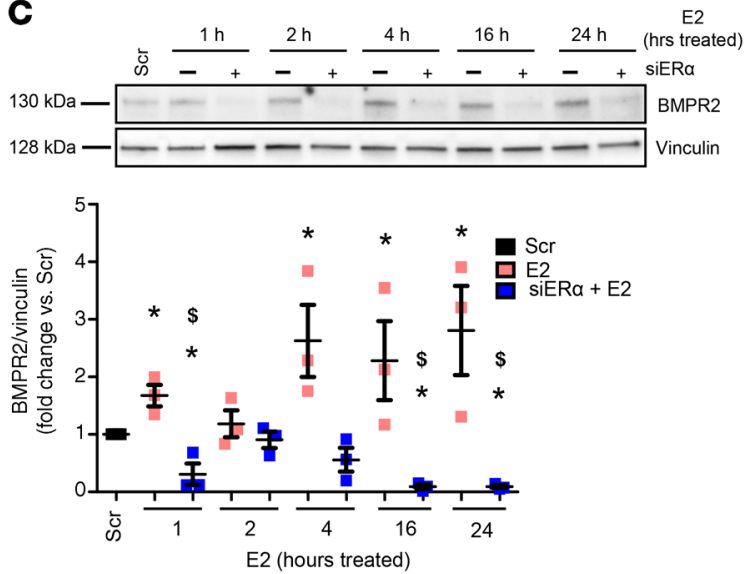

$\mathbf{F}$
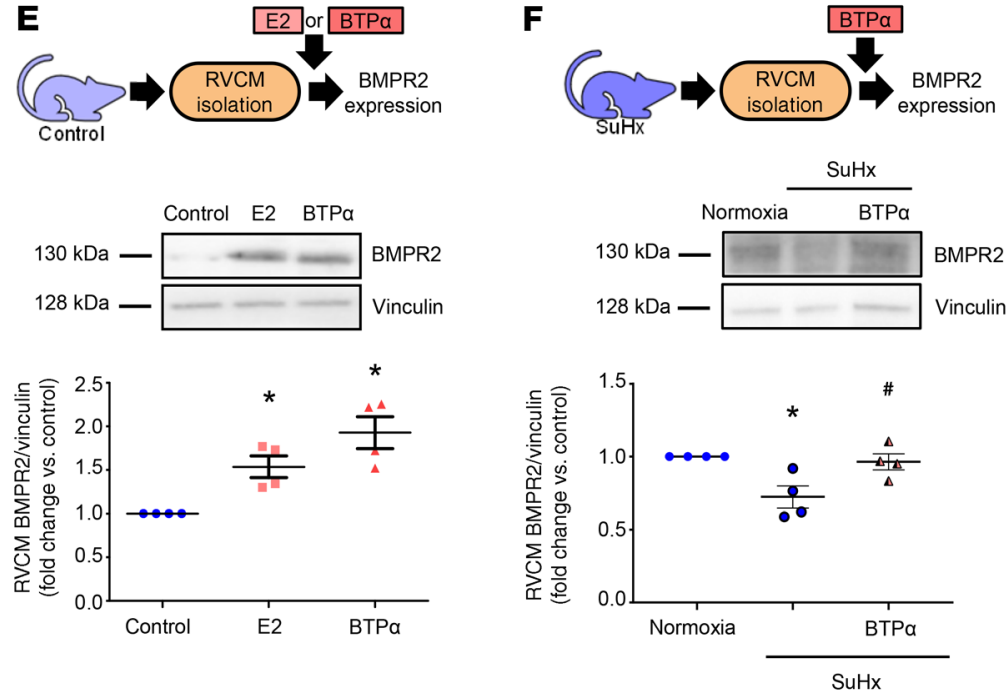

G

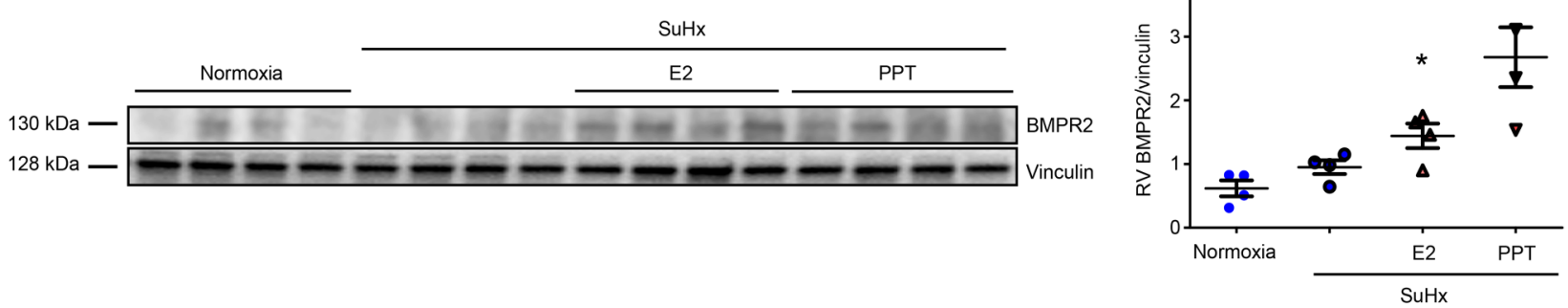

H
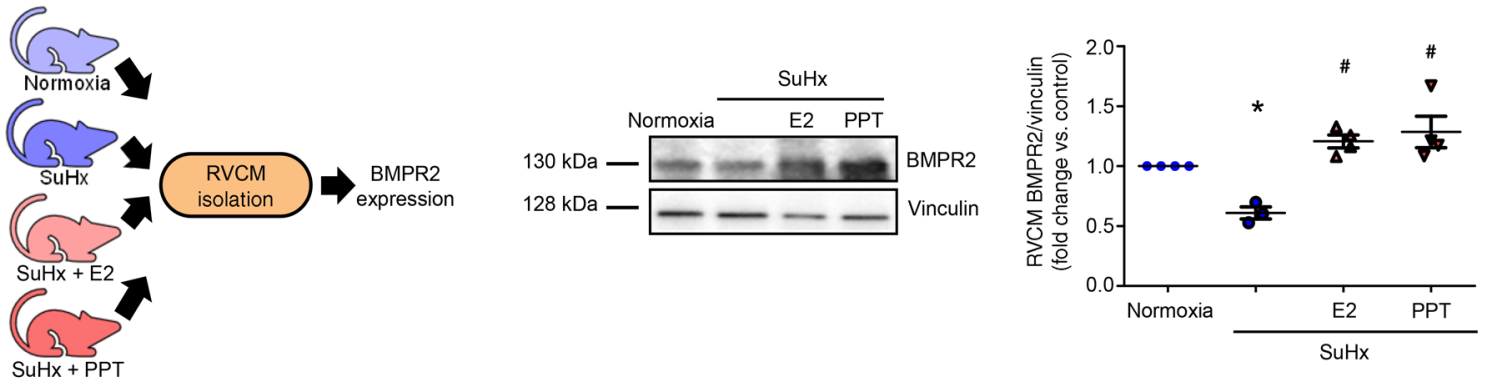
Figure 10. ER- $\alpha$ binds to Bmpr2 promoter and is necessary and sufficient to increase RV BMPR2 in vitro and in vivo. (A) RV BMPR2 and ER- $\alpha$ protein correlate positively in male and female control and SuHx-PH rats. (B) ChIP of ER- $\alpha$ binding at the Bmpr2 promoter. H9c2 cardiomyoblasts were treated with E2 (100 $\mathrm{nM}$ ) for 0 (control), 4, or 20 hours. DNA/protein complexes were cross-linked and immunoprecipitated with anti-ER- $\alpha$ antibody or IgG isotype control. RNA polymerase II (RNA Pol II) binding to Gapdh promoter was used as positive control (bottom panel). NTC: no template control. (C) Time course of BMPR2 expression in H9c2 cardiomyoblasts after ER- $\alpha$ siRNA knockdown ( $5 \mathrm{nM}$; 24 hours prior to E2 [100 $\mathrm{nM}$ ]; see Figure 4B for ER- $\alpha$ knockdown efficacy). (D) BMPR2 protein in H9c2 cells treated with ER- $\alpha$ agonist BTP- $\alpha$ (100 nM, 24 hours). (E) BMPR2 protein in RV cardiomyocytes (RVCMs) isolated from male rats and treated with E2 (10 nM, 24 hours) or BTP- $\alpha$ (100 nM, 24 hours) in vitro. (F) BMPR2 protein in RVCMs isolated from male and female SuHx-PH rats and treated in vitro with BTP- $\alpha(100 \mathrm{nM}, 24$ hours). (C) BMPR2 expression in $\mathrm{RV}$ homogenates from male normoxia, SuHx-PH, or SuHx-PH rats treated with E2 or ER- $\alpha$ agonist PPT (75 or $850 \mu \mathrm{g} / \mathrm{kg} /$ day; s.c. pellets). (H) BMPR2 protein in RVCMs from groups shown in $\mathbf{F} . n=3$ independent experiments in $\mathbf{B}-\mathbf{D} ; n=4$ rats/group in E-H. B-G depict representative Western blots. Densitometries include data from all experiments or animals. Pearson's $R$ value and $P$ value shown in $\mathbf{A}$. Dashed lines represent $95 \%$ Cl. ${ }^{*} P<0.05$ versus $\mathrm{Scr}$ (scrambled control), ${ }^{\$} P<0.05$ versus E2 in $\mathbf{C}$; ${ }^{*} P<0.05$ versus control or normoxia in $\mathbf{D}-\mathbf{H} ;{ }^{\#} P<0.05$ versus untreated SuHx in $\mathbf{F}$ and $\mathbf{H}$. $P$ values in B, C, and E-H by ANOVA/post hoc Tukey correction; $P$ value in D by Student's $t$ test. Error bars represent mean \pm SEM; each data point represents 1 experiment/animal.

repletion, and correlates with markers of RV adaptation. RV ER- $\beta$ expression, on the other hand, was not affected by sex, $\mathrm{PH}$, or hormone manipulation. In this study, we demonstrated that E2 and ER- $\alpha$ increased or maintained BMPR2 and apelin in stressed cardiomyoblasts, in isolated RVCMs, and in RVs from multiple animal models. We also provide evidence that the ER- $\alpha$ /apelin signaling axis is active in human RV tissues (Figures 1 and 2). We demonstrated that ER- $\alpha$ is necessary and sufficient to upregulate BMPR2 and apelin (Figures 6, 7, and 10) and necessary for E2 to mediate RV protection (Figure 8). We showed that ER- $\alpha$ binds to the Bmpr2 promoter in presence of E2 and that BMPR2 is necessary for RV-protective effects of E2 and for E2-mediated upregulation of apelin (Figures 10 and 11). We demonstrated functionally relevant effects of RVCM apelin (Figure 4) and established that apelin is necessary for E2 to exert RV-protective effects (Supplemental Figure 6). In addition, we identified several other downstream targets of E2 and ER- $\alpha$ (ERK1/2, P38MAPK, caspase-3/7; Supplemental Figures 5, 9, and 10). We showed that treatment with E2 or ER- $\alpha$ agonist restores RV apelin and BMPR2 and improves RV structure and function even in established $\mathrm{PH}$ (Figures 12 and 13). Importantly, E2's RV-protective effects were associated with improved survival (Supplemental Figure 15).

E2-mediated protection was observed in several models of RV pressure overload. Our studies in MCT-PH and SuHx-PH, although not allowing dissection of RV-specific effects from indirect effects mediated by changes in RV afterload, evaluated "net" effects of E2 and ER- $\alpha$ in the entire cardiopulmonary axis. They therefore provide clinically relevant information and identify exogenous E2 and ER- $\alpha$ activation as potential therapeutic strategies to attenuate pulmonary vascular and RV dysfunction. Our PAB experiments and studies in isolated RVCMs, on the other hand, demonstrated that E2 and ER- $\alpha$ indeed exert direct effects on the RV, opening a potential avenue for RV-directed therapies. The signaling pathway described here is of particular interest since it is therapeutically targetable: activators of ER- $\alpha$ (28), BMPR2 (34), or apelin (14) already are available and could rapidly be tested in clinical trials.

Our studies employing delayed treatment strategies in MCT-PH and PAB (Figures 12 and 13) suggest that E2 can maintain RV adaptation and prevent or prolong the transition to maladaptive RV hypertrophy. Although it is puzzling that E2 improved survival in PAB rats without statistically increasing cardiac function, we speculate that the increases in the SVI and cardiac index, though not statistically significant, were sufficient to positively affect survival. Treatment at an earlier time point may have been more efficacious.

ER- $\alpha$ is cardioprotective in the LV (35), and loss-of-function mutations in ESR1 have been linked to myocardial infarction and stroke $(36,37)$. However, the role of ER- $\alpha$ in the RV had not been studied. Importantly, studies from the LV cannot simply be extrapolated to the RV because both chambers are embryologically, structurally, and physiologically distinct (38). We localized ER- $\alpha$ to cardiomyocytes and demonstrated that ER- $\alpha$ increases BMPR2 and apelin and attenuates proapoptotic signaling in these cells. Colocalization of ER- $\alpha$ staining with nuclei (Figure 1A) and binding of ER- $\alpha$ to the Bmpr 2 promoter suggest that ER- $\alpha$ signals in the RV via genomic mechanisms; however, effects on apelin and BMPR2 were noted within 4 hours, suggesting that nongenomic mechanisms may occur as well. Effects of ER- $\alpha$ may extend beyond cardiomyocytes. In the LV, ER- $\alpha$ promotes angiogenesis (39). We detected ER- $\alpha$ in RVECs, and studies deciphering the role of ER- $\alpha$ in RV angiogenesis are currently underway.

ER- $\alpha$ has previously been linked to PH development (40, 41 ; however, these studies only investigated pulmonary artery smooth muscle cells and mice with chronic hypoxia (a less robust PH model), thus limiting their generalizability. Increased ER- $\alpha$ RNA and ESR1 SNPs have been associated with PAH development in humans $(42,43)$, but these studies do not allow for conclusions regarding ER- $\alpha$ 's function. Our studies demonstrated beneficial effects of ER- $\alpha$ in the RV. We previously demonstrated that E2 and ER- $\alpha$ agonist treatment improve RV function in SuHx-PH without worsening pulmonary artery remodeling (12, 13), and we have now shown that both compounds also rescue established $\mathrm{PH}$ and demonstrate beneficial effects on survival. We posit that continuously administering exogenous E2 (especially in absence of endogenous female sex hormones, as seen in ovariectomized or in male animals) or selectively activating ER- $\alpha$ are strategies to harness RV-protective effects of estrogenic signaling in PAH without negatively affecting pulmonary artery remodeling. Continuous E2 administration may be superior to endogenous release because it avoids fluctuations in levels, which may be associated with negative effects in the PA (40). This could also explain why aromatase inhibition may be beneficial in PAH $(41,44)$. However, concern exists that aromatase inhibition negatively affects RV function (45). We posit that administering exogenous E2 or ER- $\alpha$ agonist in context of a "low female sex steroid environment" (e.g., in postmenopausal female or male patients or in premenopausal females after inhibiting endogenous hormones) is safer and more efficacious than inhibiting aromatase, but this hypothesis remains to be tested. 
A

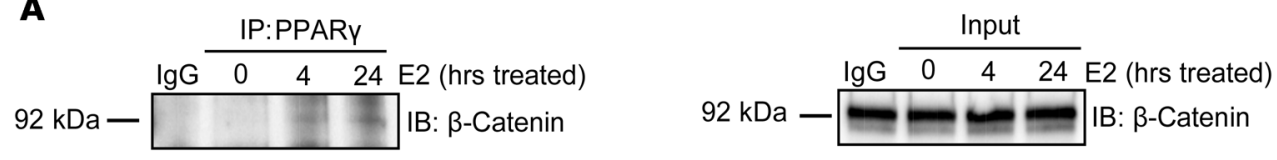

B

$$
\begin{aligned}
& \text { ì } \frac{1 \mathrm{~h}}{-++} \frac{2 \mathrm{~h}}{-+} \frac{4 \mathrm{~h}}{-+} \frac{16 \mathrm{~h}}{-+} \frac{24 \mathrm{~h}}{-+} \text { E2 (hrs treated) } \\
& 130 \mathrm{kDa}-\ldots-\ldots \text { - }- \text { BMPR2 } \\
& 17 \mathrm{kDa}--\div-2 \quad \text { Apelin } \\
& 66 \mathrm{kDa}-\ldots-\ldots-\ldots-\ldots \text { ERa }
\end{aligned}
$$

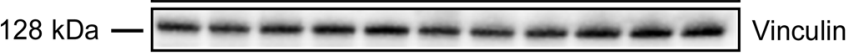

- Scr

ㅁ2

$\square$ siBMPR2 + E2
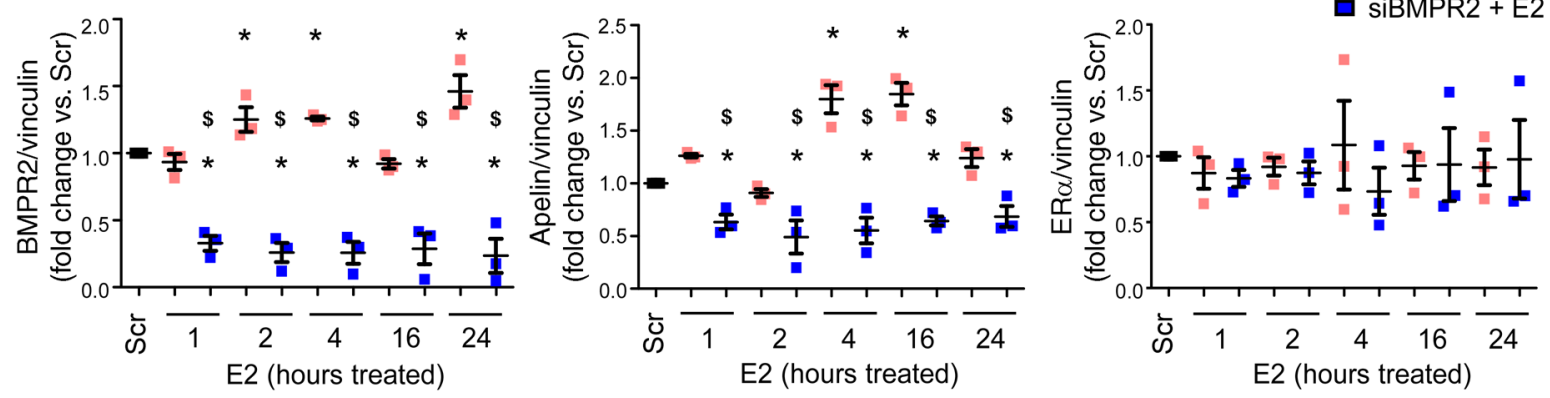

C
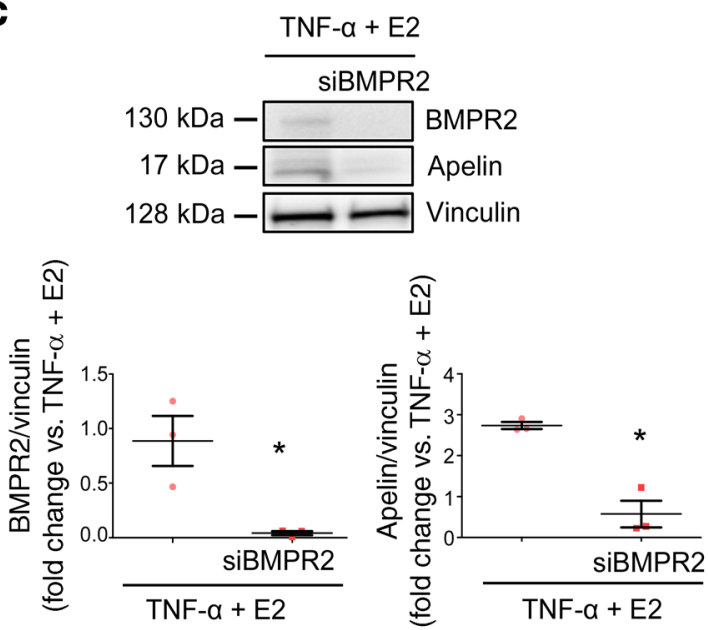

D

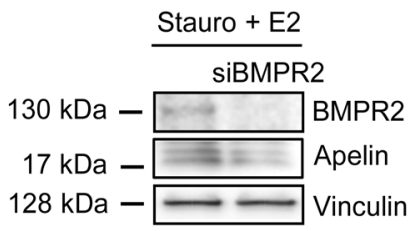

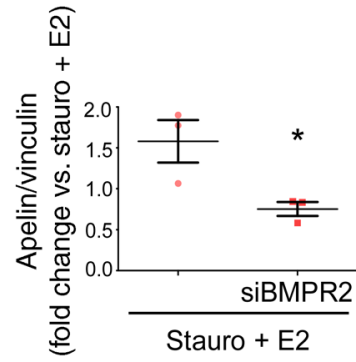
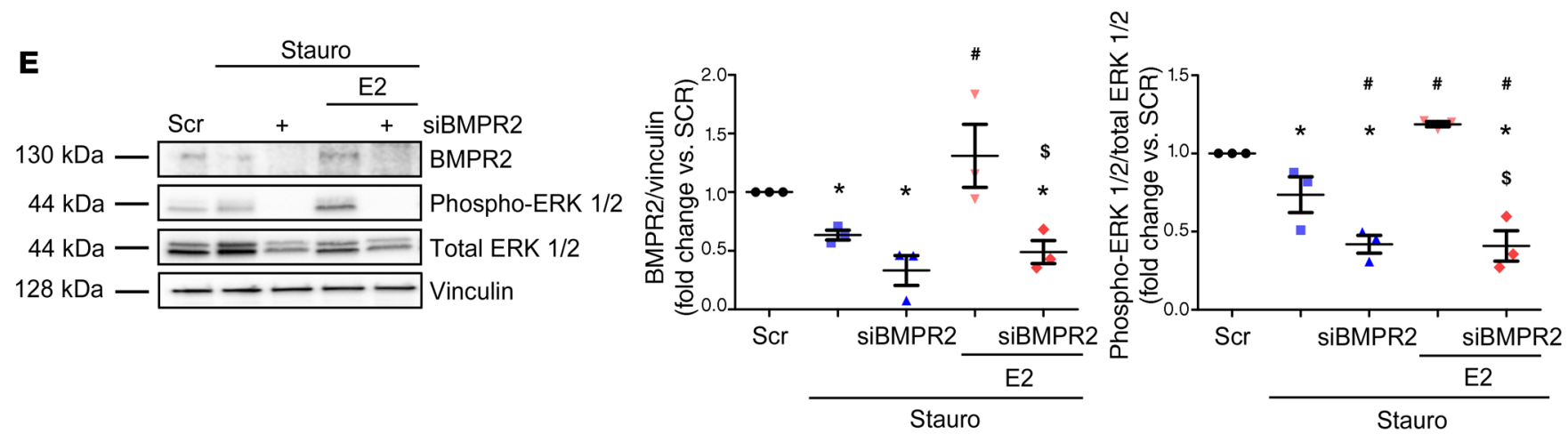
Figure 11. E2 induces formation of PPAR $\gamma / \beta$-catenin complexes and requires BMPR2 to increase apelin expression or ERK1/2 activation in cardiomyoblasts. (A) H9c2 cardiomyoblasts were treated with E2 $(100 \mathrm{nM})$ for 0,4 , or 24 hours and then immunoprecipitated with PPAR $\gamma$ antibody or rabbit IgG isotype control. Input control for each sample is indicated (bottom). Note formation of PPAR $\gamma / \beta$-catenin complexes with E2 treatment at 4 and 24 hours. (B) Time course of siRNA knockdown of BMPR2 ( $5 \mathrm{nM}$ ) effects on apelin protein expression in E2-treated (100 nM) H9c2 rat cardiomyoblasts at baseline conditions. (C and D) Effects of BMPR2 knockdown on E2-mediated upregulation of apelin in stressed cardiomyoblasts. H9c2 cells were pretreated with E2 (100 nM, 24 hours) and then stressed with TNF- $\alpha(10 \mathrm{ng} / \mathrm{ml}, 8$ hours; C) or staurosporine (stauro; $50 \mathrm{nM}, 4$ hours; D) with or without siRNA knockdown of BMPR2 ( $5 \mathrm{nM}, 24$ hours prior to E2). (E) Effects of BMPR2 knockdown on E2mediated ERK1/2 activation in stressed cardiomyoblasts. Cells were pretreated with E2 (100 nM, 24 hours) and then treated with stauro ( $50 \mathrm{nM}, 4$ hours) with or without siRNA directed against BMPR2 ( $5 \mathrm{nM} ; 24$ hours prior to $E 2$ and stauro). Representative blots for 3 independent experiments shown in $\mathbf{A}$ and $\mathbf{B}-\mathbf{E}$. Densitometries include data from all experiments. $\mathrm{Scr}=$ scramble siRNA. ${ }^{*} P<0.05$ versus Scr control, ${ }^{\$} P<0.05$ versus E2 by ANOVA with post hoc Tukey's correction in $\mathbf{B} ;{ }^{*} P<0.05$ versus TNF + E2 or Stauro + E2 by Student's $t$ test in $\mathbf{C}$ and $\mathbf{D}$; ${ }^{*} P<0.05$ versus Scr control, ${ }^{\#} P<0.05$ versus Stauro, ${ }^{\$} P<0.05$ versus E2-treated Stauro group by ANOVA with post hoc Dunnett's correction in $\mathbf{E}$. Error bars represent mean \pm SEM; each data point represents 1 experiment or animal.

Importantly, ER- $\alpha$-selective agonist treatment confers cardiovascular protection without unwanted uterotrophic effects (46).

While estrogens affect clinical outcomes in $\mathrm{PAH}$, and while female sex is associated with a survival benefit in this disease, additional factors such as other sex hormones and age also are modifiers of outcomes (47). This may explain why the survival difference between male and female patients in the REVEAL cohort is driven by males older than 60 years (48). Interactions between sex and age and the distinct patient population enrolled in REVEAL may explain the specific observations in this cohort. REVEAL also demonstrated a higher female/male ratio compared with other registries, suggesting that patients in this cohort may be distinct from other PAH populations (48). This may result from enrollment of individuals with higher wedge pressures or different comorbidities.

Upstream regulators and downstream targets of BMPR2 in the RV are incompletely understood. In the lung, BMPR2 induces apelin (29), and decreased activation of the BMPR2/apelin axis causes PAH (29). Although the role of BMPR2 signaling in RVF had been unstudied until recently, evolving evidence suggests relevant RV effects. First, absence of BMPR2 during cardiac development causes RV outflow tract malformations $(17,18)$. Second, PAH patients with $B M P R 2$ mutations exhibit more profound RVF than patients without a mutation (49). Third, BMPR2-mutant cardiomyoblasts and RVs from BMPR2-mutant mice exhibit abnormalities in fatty acid oxidation (50) as well as calcium signaling and cell contractility (31). We have expanded the current knowledge by identifying BMPR2 as a target of E2/ER- $\alpha$ in RVCMs. Binding of ER- $\alpha$ to the Bmpr 2 promoter and formation of $\beta$-catenin/PPAR $\gamma$ complexes after $\mathrm{E} 2$ treatment suggest that E2, via ER- $\alpha$, increases BMPR2 and that BMPR2, via $\beta$-catenin/PPAR $\gamma$, increases apelin. This does not rule out that ER- $\alpha$ directly interacts with apelin as well; however, apelin expression was profoundly decreased after BMPR2 knockdown (Figure 11B). Since E2 treatment resulted in $\beta$-catenin/PPAR $\gamma$ complex formation and given the recently observed RV-protective effects of PPAR $\gamma$ activation (51), it is also conceivable that stimulatory effects on PPAR $\gamma$ signaling and fatty acid oxidation contribute to E2's RV protection. Our data suggest that E2/ER- $\alpha$ may also employ canonical BMPR2 signaling (Supplemental Figures 11 and 12). Ongoing investigations are evaluating how BMPR2 expression and signaling are decreased in RVF. Mechanisms could include decreased BMPR2 synthesis, increased BMPR2 turnover, and/or upregulation of BMPR2 inhibitors. Our studies in $\triangle 71$ rats (Supplemental Figure 13) suggest that BMPR2 indeed is necessary for RV-protective effects of E2 in vivo. Future studies will evaluate time courses, different degrees of BMPR2 loss of function, and larger animal numbers.

Our findings of stimulatory effects of E2/ER- $\alpha$ on BMPR2 contradict a paradigm where E2 decreases BMPR2 expression in pulmonary artery smooth muscle cells or lymphocytes (52, 53). We believe that such a paradigm is overly simplistic. E2 is pleiotropic, and discrepancies between studies could result from dose-, context-, and compartment-specific effects. Of note, we also detected stimulatory effects of E2 and ER- $\alpha$ on BMPR2 in pulmonary artery endothelial cells (54). Studies in specific cell types are necessary to further dissect effects of estrogenic signaling on BMPR2. Our studies suggest that E2 and ER- $\alpha$ can increase BMPR2 and that the "net" effect of E2 or ER- $\alpha$ activation in experimental PH is salutary (Figures 8 and 12 and Supplemental Figure 15).

Apelin, via APLNR, exerts procontractile, proangiogenic, and antiapoptotic effects in the LV (55). Emerging data suggest a role for apelin in the RV: APLNR-KO mice exhibit dilated and/or deformed RVs, apelin-KO mice exhibit exaggerated $\mathrm{PH}$, and circulating apelin is decreased in PAH patients (56, 57). Apelin is an inotrope in the $\operatorname{RV}(15,33)$ and is decreased in SuHx-PH RVs $(12,58)$. Short-term infusion of Pyr-apelin-13 reduces pulmonary vascular resistance and increases cardiac output in PAH patients (14). Our human RV data linked apelin to less RV fibrosis and hypertrophy (Figure 2, D and E). However, mechanisms and regulators of apelin-mediated RV-protective signaling are unknown. We identified 2 previously unidentified regulators of RV apelin and provide mechanistic evidence linking apelin to better RV function.

Prior studies indicated that apelin is predominantly expressed in endothelial cells $(22,23)$, and we now provide evidence that apelin is also expressed in rat and human RVCMs (Figures 2, F and G, 3D), that apelin is decreased in RVCMs from RVF patients (Figure 2F), and that RVCM-derived apelin exerts paracrine and potentially autocrine effects on proangiogenic effects in RVECsas well as stimulatory effects on procontractile and prosurvival signaling in RVCMs (Figure 4 and Supplemental Figure 4). Interestingly, recent studies employing single-cell RNA-Seq in human or mouse hearts also demonstrated that apelin is expressed in several cardiomyocyte populations $(59,60)$. Our data suggest that the presence of apelin may not be limited to endothelial cells and that RVCM apelin may also exert physiologically relevant effects. Studies of ER- $\alpha$ /BMPR2/apelin signaling in other cell types, such as RVECs or pulmonary artery endothelial cells, are currently underway.

Our study has limitations. First, H9c2 cardiomyoblasts are not RV specific. However, we confirmed results obtained in these 


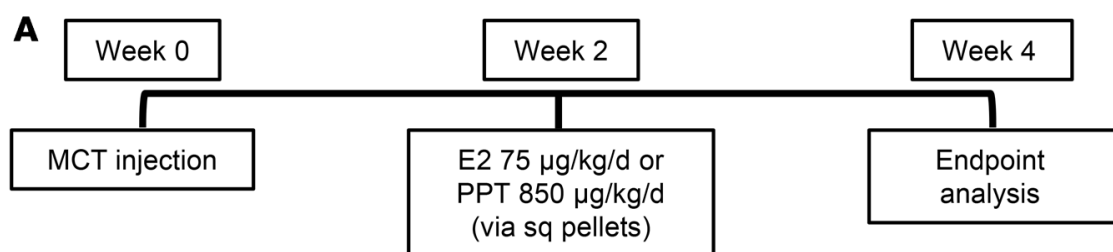

B
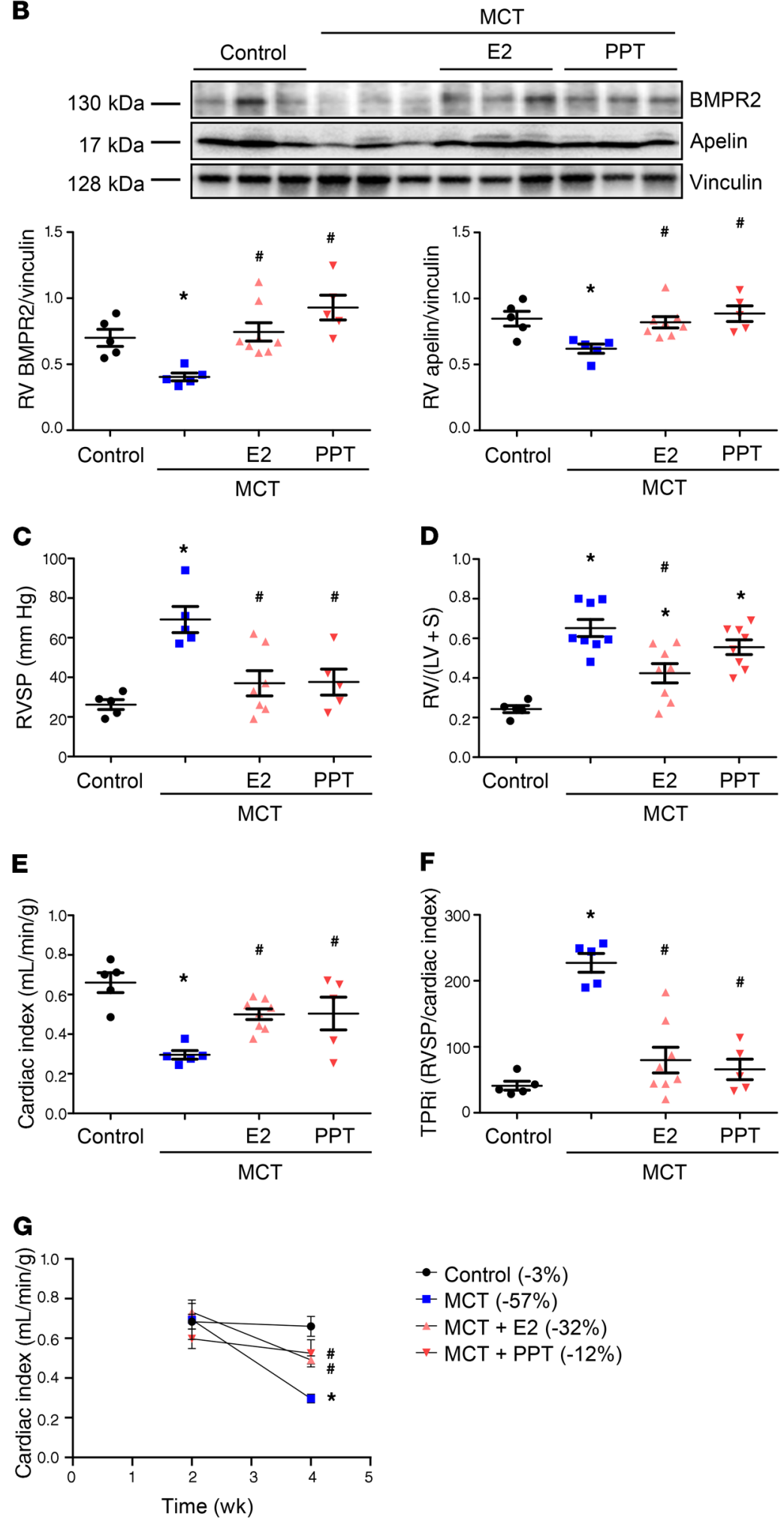

cells in vivo and in isolated RVCMs. This suggests that, given the
Figure 12. E2 or ER- $\alpha$ agonist PPT rescues MCTinduced PH and increases RV BMPR2 and apelin.

(A) Experimental design. (B) Western blot analysis of RVs from MCT-PH rats. A representative Western blot is depicted on top of panel; densitometric analysis from all experimental animals is shown at bottom. (C-F) Effects of E2 or PPT on RV systolic pressure (RVSP; C), RV hypertrophy (RV weight divided by weight of left ventricle plus septum; $\mathrm{RV} /[\mathrm{LV}+\mathrm{S}] ; \mathbf{D})$, cardiac index (echocardiographic cardiac output/body weight; E), and total pulmonary resistance index (cardiac index/RVSP; F). (G) Time course of cardiac index. Percentage change in cardiac index versus 2-week baseline time point is shown in legend in parentheses behind group names. ${ }^{*} P<0.05$ versus control, ${ }^{\#} P<0.05$ versus untreated MCT (1-way ANOVA with Tukey or Dunnett's post hoc correction). Each data point in B-F represents 1 male animal. Error bars represent mean $\pm \mathrm{SEM} . \mathrm{Sq}=$ subcutaneous.

limitations of primary cardiomyocyte culture, $\mathrm{H} 9 \mathrm{c} 2$ cells are appropriate for mechanistic studies, as long as results are corroborated in cardiomyocytes and/or in vivo. Second, studies in human RV tissue were limited by lack of tissue from disease-free controls and lack of cardiac output data from all individuals. Differences between RVF patients and healthy controls may be more pronounced; however, RV tissue from healthy subjects is not available. Third, apelin loss-of-function studies employed pharmacological blockade of APLNR rather than genetic manipulation. However, studies in transgenic animals would have required mice, which would be limited by lack of a robust RVF phenotype (3). Experiments using a rat model therefore are more clinically relevant. Future studies will specifically evaluate the role of cardiomyocyte apelin in vivo (along with cardiomyocyte ER- $\alpha$ and BMPR2). Fourth, while we employed several methods to determine that apelin is expressed and functionally relevant in RVCMs, and while apelin expression was identified through single-cell RNA-Seq (59, 60 ), the most definite method to establish a functional role of RVCM apelin would be through development of an RVCM-specific loss-of-function rodent model. We will focus on such studies in the future. Finally, correlations between ER- $\alpha$ and apelin expression in human RV could only be established in RVF patients. Interestingly, ER- $\alpha$ expression in control hearts was more variable. This could be due to the patients' sex, age, or other unknown modifiers. However, the ER- $\alpha$ /apelin correlation during RVF was 
A
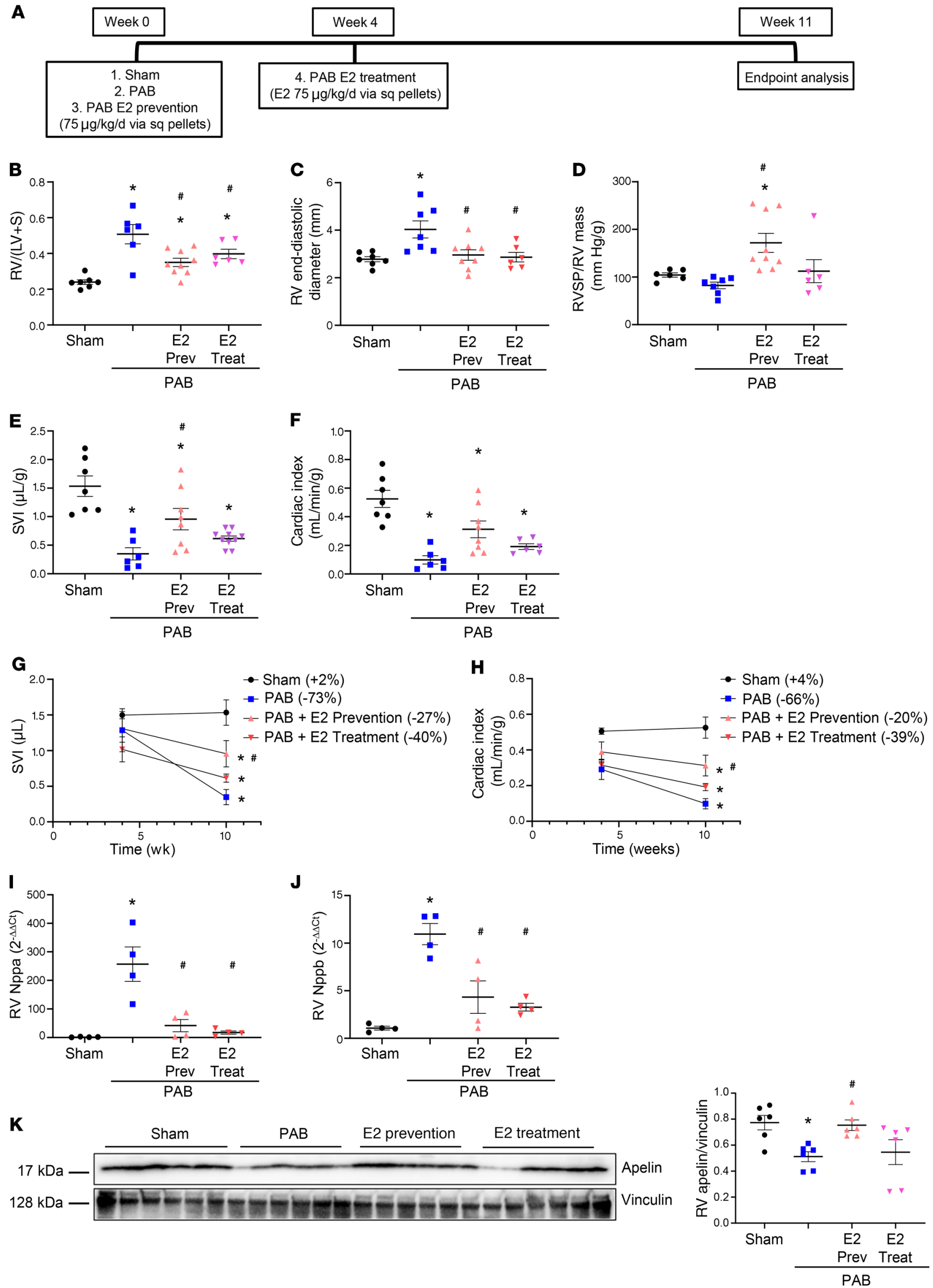
Figure 13. E2 prevents and rescues RV failure induced by pulmonary artery banding. (A) Experimental design. (B-H) Effects of E2 treatment on RV hypertrophy ( $R V$ weight divided by weight of left ventricle plus septum; $\mathrm{RV} /[\mathrm{LV}+\mathrm{S}]$; B), RV end-diastolic diameter (C), RV systolic pressure (RVSP) normalized for RV mass (D), stroke volume index (SVI; E) and cardiac index (F). SVI and cardiac index were determined echocardiographically. (G-H) Time courses of SVI and cardiac index. Percentage change in SVI and cardiac index versus 4-week baseline time point is shown in legends in parentheses behind group names. (I-J) Effects of E2 treatment on atrial natriuretic peptide (Nppa, I) and B-type natriuretic peptide (Nppb, J) by real time RT-PCR. (K) Quantification of RV apelin by Western blot and densitometric analysis. ${ }^{*} P<0.05$ versus sham control, ${ }^{\#} P<0.05$ versus untreated PAB (1-way ANOVA with Tukey's or Dunnett's post hoc correction). Each data point in B-F and $\mathbf{I - K}$ represents 1 male animal. Error bars represent mean \pm SEM. Sq = subcutaneous; Prev = prevention group; Treat $=$ treatment group.

robust and corroborated in several animal models. Although hemodynamic or echocardiographic data were not available for all subjects, occurrence of RVF was clinically adjudicated and confirmed at autopsy.

In summary, we identified a molecular mechanism of E2 and ER- $\alpha$-mediated attenuation of RVF in PAH. We identified BMPR2 and apelin as regulators of prosurvival and adaptive signaling in the RV and as targets of E2 and ER- $\alpha$. Harnessing this cardioprotective E2/ER- $\alpha$ /BMPR2/apelin axis may allow for developing nonhormonal and RV-directed therapies for PAH patients of either sex.

\section{Methods}

\section{General}

All experiments were performed in accordance with recent recommendations $(3,61)$, including randomization and blinding at the time of measurement and analysis.

\section{Animal models}

SuHx-PH. Su5416 (20 mg/kg s.c.; dissolved in DMSO; Sigma-Aldrich) was administered immediately prior to hypoxia exposure. Hypoxia exposure occurred in a hypobaric chamber for 3 weeks, followed by room air exposure for 4 weeks (12). Experimental groups included intact male and female age-matched Sprague-Dawley normoxic controls, intact male and female SuHx-PH rats, ovariectomized (OVX; described previously, ref. 12) SuHx-PH females, and OVX SuHx-PH females replete with E2 $(75 \mu \mathrm{g} / \mathrm{kg} / \mathrm{d}$ via s.c. pellets, resulting in physiological levels, ref. 12; Innovative Research of America). Males weighed 175 to $200 \mathrm{~g}$; females weighed 150-175 g (Charles River Laboratories). Additional studies were performed in male SuHx-PH rats with or without E2 (75 $\mu \mathrm{g} / \mathrm{kg} /$ day; s.c. pellets) or ER- $\alpha$-selective agonist PPT (850 $\mu \mathrm{g} / \mathrm{kg} /$ day; s.c. pellets, ref. 12). Vehicle for E2 or PPT did not have any effects on cardiopulmonary parameters in prior studies and was not included in the current analysis (62).

Hypoxia-induced $\mathrm{PH}$. Male and female age-matched Sprague-Dawley rats containing loss-of-function mutations in Esr1 (encoding ER- $\alpha$ ) and their WT littermate controls were exposed to 3 weeks of normobaric hypoxia (equivalent to $10 \% \mathrm{O}_{2}$ ). Experimental detail and validation of Esr1 mutations are found in Supplemental Table 1 and Supplemental Figure 16. In addition, male WT, Esr1 ${ }^{-/}$, and Esr $2^{-/}$(encoding ER- $\beta$ ) C57BL/6 mice (20-22 g; The Jackson Laboratory) were exposed to 3 weeks of hypobaric hypoxia while treated with E2 $(75 \mu \mathrm{g} / \mathrm{kg} / \mathrm{d}$; s.c. pellets).

MCT-PH. Male Sprague-Dawley rats (200-250 g) were s.c. injected with MCT (60 mg/kg; Sigma-Aldrich). Two weeks later, subgroups of animals received E2 (75 $\mu \mathrm{g} / \mathrm{kg} /$ day) or PPT ( $850 \mu \mathrm{g} / \mathrm{kg} /$ day $)$ for 2 more weeks via s.c. pellets, followed by endpoint analysis.

$P A B$. Male Sprague-Dawley rats (150-180 g) were anesthetized by inhaled isoflurane and orotracheally intubated. A small incision was made through the intercostal muscle and extended toward the sternum and the spine, and the thoracic cavity opened. The PA was then dissected, and a 4-0 silk suture was passed around the PA and constricted using a $19 \mathrm{G}$ needle hub. The thoracic cavity was then closed, reevacuated, and monitored for refilling of air, and muscle layers sutured. Sham controls underwent an identical surgical procedure without PA constriction. Additional studies were performed in male $\mathrm{PAB}$ rats with E2 (75 $\mu \mathrm{g} / \mathrm{kg} /$ day; s.c. pellets) given at the time of surgery (preventative group) or 4 weeks after surgery, when cardiac output began to decrease (treatment group).

\section{RNA-Seq of bovine RV}

RNA-Seq was performed in RV tissue from yearling steers and data deposited in the NCBI Gene Expression Omnibus (GEO, GSE164320). See Supplemental Methods for details on experiments.

\section{Human RV tissue}

Human RV free wall tissue was collected as described previously (21, 63) from warm autopsies ( $<3$ hours after death) or cardiac surgery. The RVF group consisted of patients with congenital heart disease without LV involvement and patients with idiopathic or scleroderma-associated end-stage PAH (age $49.9 \pm 5.5$ years; $70 \%$ female). RVF was defined as decreased tricuspid annular plane systolic excursion and/or death from RVF (21). The presence of RVF was confirmed during autopsy. Control group tissues were obtained from donors with coronary artery disease or aortic stenosis without evidence of PAH or RV hypertrophy by echocardiography or histological analysis (refs. 21,$63 ; 45.1 \pm 3.8$ years; $77 \%$ female). Clinical characteristics are described in Supplemental Table 2.

\section{RVCM isolation}

RVCMs were isolated using the Adult Rat/Mouse Cardiomyocyte Isolation Kit (Cellutron Life Technologies). Hearts were perfused via a simplified Langendorff system and Cole-Parmer MasterFlex peristaltic pump at $8 \mathrm{ml} / \mathrm{min}$ per the manufacturer's guidelines. After perfusion, the RV was dissected and digested. Approximately $3 \times 10^{5}$ viable RVCMs (Supplemental Figure 17) were seeded in a laminin-coated 6-well plate and maintained in adult cardiomyocyte medium with serum (Cellutron Life Technologies). Cells were maintained for at least 6 hours after isolation prior to treatment. Experiments were performed within 24 hours after isolation.

\section{Statistics}

Results are expressed as mean \pm SEM. At least 3 biologically independent experiments (run in technical duplicates) were performed for all in vitro studies and reported as $N$. Statistical analyses were performed with GraphPad Prism 6. Sample sizes were estimated by power calculation. A 2-tailed Student's $t$ test or 1-way ANOVA with Tukey's or Dunnett's post hoc correction was used for comparison 
of experimental groups. Correlations were determined using Pearson's coefficient $(R)$. Statistically significant difference was accepted at $P$ less than 0.05 .

\section{Study approval}

All human studies were approved by and performed in accordance with the Laval University and Institute Universitaire de Cardiologie et de Pneumologie de Québec biosafety and human ethics committees (CER-20773). All patients or their legal representatives (in case of autopsy) gave informed consent before beginning of the study. All rodent experiments were approved by the Indiana University School of Medicine or Laval University IACUCs (protocols 11220 and 2018-015-2) and adhered to NIH guidelines for care and use of laboratory animals.

See Supplemental Data for additional methods.

\section{Author contributions}

ALF designed, performed, and analyzed studies and wrote the manuscript; MA, BY, and BR performed experiments; VN, ET, SBB, $\mathrm{FP}$, and JO performed and analyzed experiments and provided data; ET, SB, AF, ALF, and TC performed hemodynamic and echocardiographic experiments; RDB and KRS performed and provided bovine RNA-Seq data; CDR, KK, RL, XS, NCC, and TL generated ER- $\alpha$ mutant rats; DMT and NCC conducted hypoxic ER- $\alpha$ mutant rat studies; SP and SB provided RV tissue, designed and analyzed experiments, and edited the manuscript; TL designed and analyzed studies and wrote and edited the manuscript.

\section{Acknowledgments}

We thank Robert G. Presson, Jr. for equipment support; the Indiana University School of Medicine Research Immunohistochemistry Facility for technical support; Henry Bryant and Jeffrey Dodge at Eli Lilly \& Co. for academic use of BTP- $\alpha$; Frederic Perros for providing $\Delta 71$ rats; and Chiemela Ubagharagi, Emily Seiden, Fatiha Iqbal, and Jiajun Li for technical support. AF is supported by American Heart Association (AHA) Career Development Award 19CDA34660173, American Lung Association Catalyst Award CA-629145, and Actelion Entelligence Young Investigator Award and was previously supported by an AHA postdoctoral fellowship 17POST33670365 and a Indiana Clinical Translational Sciences Institute (CTSI) postdoctoral fellowship 5TL1TR001107-02 (NIH/ NCATS). TL is supported by AHA 17GRNT33690017, Veterans Affairs Merit Review Award 2 I01 BX002042-05, National Heart, Lung, and Blood Institute (NHLBI) 1R56HL134736-01A1, and NIH HL144727-01A1. KS is supported by NHLBI PPG P01 HL014985, NHLBI R01HL114887, and Department of Defense PR140977. SB and SP are supported by Centre de Recherche Institute Universitaire de Cardiologie et de Pneumologie de Québec (CRI-UCPQ) and by the Canadian Institutes of Health Research. SB is supported by the Heart and Stroke Foundation of Canada.

Address correspondence to: Tim Lahm, Walther Hall, C400, 980 W. Walnut St, Indianapolis, Indiana 46202, USA. Phone: 317.278.0413; Email: tlahm@iu.edu. RL and XS's present address is: University of California, San Diego, California, USA.
1. Rabinovitch M. Molecular pathogenesis of pulmonary arterial hypertension. J Clin Invest. 2012;122(12):4306-4313.

2. Lajoie AC, et al. Combination therapy versus monotherapy for pulmonary arterial hypertension: a meta-analysis. Lancet Respir Med. 2016;4(4):291-305.

3. Lahm T, et al. Assessment of right ventricular function in the research setting: knowledge gaps and pathways forward. An official American Thoracic Society research statement. Am J Respir Crit Care Med. 2018;198(4):e15-e43.

4. Vonk Noordegraaf A, et al. Pathophysiology of the right ventricle and of the pulmonary circulation in pulmonary hypertension: an update. Eur Respir J. 2019;53(1):1801900.

5. Humbert M, et al. Survival in incident and prevalent cohorts of patients with pulmonary arterial hypertension. Eur Respir J. 2010;36(3):549-555.

6. Lahm T, et al. Progress in solving the sex hormone paradox in pulmonary hypertension. Am J Physiol Lung Cell Mol Physiol. 2014;307(1):L7-26.

7. Ventetuolo CE, et al. Sex hormones are associated with right ventricular structure and function: the MESA-right ventricle study. Am J Respir Crit Care Med.2011;183(5):659-667.

8. Jacobs $W$, et al. The right ventricle explains sex differences in survival in idiopathic pulmonary arterial hypertension. Chest. 2014;145(6):1230-1236.

9. Swift AJ, et al. Right ventricular sex differences in patients with idiopathic pulmonary arterial hypertension characterised by magnetic resonance imaging: pair-matched case controlled study. PLoS One. 2015;10(5):e0127415

10. Tello K, et al. Sex differences in right ventricularpulmonary arterial coupling in pulmonary arterial hypertension. Am J Respir Crit Care Med. 2020;202(7):1042-1046.

11. Ventetuolo CE, et al. Sex and hemodynamics in pulmonary arterial hypertension. Eur Respir J. 2014;43(2):523-530.

12. Frump AL, et al. Estradiol improves right ventricular function in rats with severe angioproliferative pulmonary hypertension: effects of endogenous and exogenous sex hormones. Am J Physiol Lung Cell Mol Physiol. 2015;308(9):L873-890.

13. Lahm T, et al. $17 \beta$-Estradiol mediates superior adaptation of right ventricular function to acute strenuous exercise in female rats with severe pulmonary hypertension. Am J Physiol Lung Cell Mol Physiol. 2016;311(2):L375-L388.

14. Brash L, et al. Short-term hemodynamic effects of apelin in patients with pulmonary arterial hypertension. JACC Basic Transl Sci. 2018;3(2):176-186.

15. Dai T, et al. Apelin increases contractility in failing cardiac muscle. Eur J Pharmacol. 2006;553(1-3):222-228.

16. Szokodi I, et al. Apelin, the novel endogenous ligand of the orphan receptor APJ, regulates cardiac contractility. Circ Res. 2002;91(5):434-440.

17. Delot EC, et al. BMP signaling is required for septation of the outflow tract of the mammalian heart. Development. 2003;130(1):209-220.

18. Beppu H, et al. BMP type II receptor regulates positioning of outflow tract and remodeling of atrioventricular cushion during cardiogenesis.
Dev Biol. 2009;331(2):167-175.

19. Kidoya H, Takakura N. Biology of the apelinAPJ axis in vascular formation. J Biochem. 2012;152(2):125-131.

20. Austin ED, et al. Gender, sex hormones and pulmonary hypertension. Pulm Circ. 2013;3(2):294-314.

21. Potus F, et al. Downregulation of microRNA-126 contributes to the failing right ventricle in pulmonary arterial hypertension. Circulation. 2015;132(10):932-943.

22. Liu Q, et al. Genetic targeting of sprouting angiogenesis using Apln-CreER. Nat Commun. 2015;6:6020

23. Sheikh AY, et al. In vivo genetic profiling and cellular localization of apelin reveals a hypoxiasensitive, endothelial-centered pathway activated in ischemic heart failure. Am J Physiol Heart Circ Physiol. 2008;294(1):H88-H98.

24. Bai B, et al. Apelin-13 induces ERK1/2 but not p38 MAPK activation through coupling of the human apelin receptor to the Gi2 pathway. Acta Biochim Biophys Sin (Shanghai). 2008;40(4):311-318.

25. Perjés Á, et al. Apelin increases cardiac contractility via protein kinase $\mathrm{C} \varepsilon$ - and extracellular signal-regulated kinase-dependent mechanisms. PLoS One. 2014;9(4):e93473.

26. Bogaard HJ, et al. The right ventricle under pressure: cellular and molecular mechanisms of right-heart failure in pulmonary hypertension. Chest. 2009;135(3):794-804.

27. Tuder RM, et al. Relevant issues in the pathology and pathobiology of pulmonary hypertension. J Am Coll Cardiol. 2013;62(25 Suppl):D4-12. 
28. Chalmers MJ, et al. Hydrophobic interactions improve selectivity to ER- $\alpha$ for Ben-zothiophene SERMs. ACS Med Chem Lett. 2012;3(3):207-210.

29. Alastalo TP, et al. Disruption of PPAR $\gamma / \beta$-cateninmediated regulation of apelin impairs BMPinduced mouse and human pulmonary arterial EC survival. J Clin Invest. 2011;121(9):3735-3746.

30. Bourdeau V, et al. Genome-wide identification of high-affinity estrogen response elements in human and mouse. Mol Endocrinol. 2004;18(6):1411-1427.

31. Hautefort A, et al. Bmpr2 mutant rats develop pulmonary and cardiac characteristics of pulmonary arterial hypertension. Circulation . 2019;139(7):932-948.

32. Neto-Neves EM, et al. Isolated heart model demonstrates evidence of contractile and diastolic dysfunction in right ventricles from rats with sugen/hypoxia-induced pulmonary hypertension. Physiol Rep. 2017;5(19):e13438.

33. Yang P, et al. Elabela/toddler is an endogenous agonist of the apelin APJ receptor in the adult cardiovascular system, and exogenous administration of the peptide compensates for the downregulation of its expression in pulmonary arterial hypertension. Circulation. 2017;135(12):1160-1173.

34. Long L, et al. Selective enhancement of endothelial BMPR-II with BMP9 reverses pulmonary arterial hypertension. Nat Med. 2015;21(7):777-785.

35. Murphy E. Estrogen signaling and cardiovascular disease. Circ Res. 2011;109(6):687-696.

36. Schuit SC, et al. Estrogen receptor alpha gene polymorphisms and risk of myocardial infarction. JAMA. 2004;291(24):2969-2977.

37. Ardelt AA, et al. Estradiol regulates angiopoietin-1 mRNA expression through estrogen receptoralpha in a rodent experimental stroke model. Stroke. 2005;36(2):337-341.

38. Haddad F, et al. Right ventricular function in cardiovascular disease, part I: anatomy, physiology, aging, and functional assessment of the right ventricle. Circulation. 2008;117(11):1436-1448.

39. van Rooij E, et al. Myocyte enhancer factor 2 and class II histone deacetylases control a gender-specific pathway of cardioprotection mediated by the estrogen receptor. Circ Res. 2010;106(1):155-165.

40. Wright AF, et al. Oestrogen receptor alpha in pulmonary hypertension. Cardiovasc Res. 2015;106(2):206-216

41. Mair KM, et al. Sex-dependent influence of endogenous estrogen in pulmonary hypertension. Am J Respir Crit Care Med. 2014;190(4):456-467.

42. Rajkumar R, et al. Genomewide RNA expression profiling in lung identifies distinct signatures in idiopathic pulmonary arterial hypertension and secondary pulmonary hypertension. Am J Physiol Heart Circ Physiol. 2010;298(4):H1235-H1248.

43. Roberts KE, et al. Genetic risk factors for portopulmonary hypertension in patients with advanced liver disease. Am J Respir Crit Care Med. 2009;179(9):835-842.

44. Kawut SM, et al. Anastrozole in pulmonary arterial hypertension. A randomized, double-blind, placebo-controlled trial. Am JRespir Crit Care Med. 2017;195(3):360-368.

45. Lahm T, Frump AL. Toward harnessing sex steroid signaling as a therapeutic target in pulmonary arterial hypertension. Am J Respir Crit Care Med. 2017;195(3):284-286.

46. Bolego C, et al. Selective estrogen receptor-alpha agonist provides widespread heart and vascular protection with enhanced endothelial progenitor cell mobilization in the absence of uterotrophic action. FASEB J. 2010;24(7):2262-2272.

47. Badlam JB, Austin ED. Beyond oestrogens: towards a broader evaluation of the hormone profile in pulmonary arterial hypertension. Eur Respir J. 2018;51(6):1801058.

48. Benza RL, et al. Predicting survival in pulmonary arterial hypertension. Circulation. 2010;122(2):164-172.

49. van der Bruggen CE, et al. Bone morphogenetic protein receptor type 2 mutation in pulmonary arterial hypertension: a view on the right ventricle. Circulation. 2016;133(18):1747-1760.

50. Talati MH, et al. Mechanisms of lipid accumulation in the bone morphogenetic protein receptor type 2 mutant right ventricle. Am J Respir Crit Care Med. 2016;194(6):719-728.

51. Legchenko E, et al. PPAR $\gamma$ agonist pioglitazone reverses pulmonary hypertension and prevents right heart failure via fatty acid oxidation. $\mathrm{Sci}$ Transl Med. 2018;10(438):eaao0303.

52. Austin ED, et al. BMPR2 expression is suppressed by signaling through the estrogen receptor. Biol
Sex Differ. 2012;3(1):6.

53. Mair KM, et al. Sex affects bone morphogenetic protein type II receptor signaling in pulmonary artery smooth muscle cells. Am J Respir Crit Care Med. 2015;191(6):693-703.

54. Frump AL, et al. Selective activation of estrogen receptor a stimulates pulmonary vascular homeostatic regulator apelin in pulmonary artery endothelial cells (PAECs) from patients with pulmonary arterial hypertension (PAH). Presented at the 2019 American Thoracic Society International Conference; May 17-22, 2019; Dallas, Texas, USA. https://www.atsjournals.org/doi/10.1164/ ajrccm-conference.2019.199.1_MeetingAbstracts. A7399. Accessed January 25, 2021.

55. Dalzell JR, et al. The emerging potential of the apelin-apj system in heart failure. JCard Fail. 2015;21(6):489-498.

56. Kang Y, et al. Apelin-APJ signaling is a critical regulator of endothelial MEF2 activation in cardiovascular development. Circ Res. 2013;113(1):22-31.

57. Chandra SM, et al. Disruption of the apelin-APJ system worsens hypoxia-induced pulmonary hypertension. Arterioscler Thromb Vasc Biol. 2011;31(4):814-820.

58. Drake JI, et al. Molecular signature of a right heart failure program in chronic severe pulmonary hypertension. Am J Respir Cell Mol Biol. 2011;45(6):1239-1247.

59. Hu P, et al. Single-nucleus transcriptomic survey of cell diversity and functional maturation in postnatal mammalian hearts. Genes Dev. 2018;32(19-20):1344-1357.

60. Tucker Nathan R, et al. Transcriptional and cellular diversity of the human heart. Circulation. 2020;142(5):466-482.

61. Provencher S, et al. Standards and methodological rigor in pulmonary arterial hypertension preclinical and translational research. Circ Res. 2018;122(7):1021-1032.

62. Lahm T, et al. 17 $\beta$-Estradiol attenuates hypoxic pulmonary hypertension via estrogen receptormediated effects. Am J Respir Crit Care Med. 2012;185(9):965-980.

63. Omura J, et al. Identification of the long non-coding RNA H19 as a new biomarker and therapeutic target in right ventricular failure in pulmonary arterial hypertension. Circulation. 2020;142(15):1464-1484 